\title{
Abordagem para Seleção de Variáveis Preditivas no Contexto de Controle de Inventários
}

\author{
RESUMO
}

\section{Evandro Burgel}

eburgel0512@gmail.com

Rio Grande do Sul (UFRGS), Porto Alegre, RS, Brasil

\section{Michel José Anzanello}

anzanello@producao.ufrgs.br

Universidade Federal do Rio Grande do Sul (UFRGS), Porto Alegre, RS, Brasil
A obsolescência de estoques é um fenômeno de destaque nas organizações, demandando o uso de métodos que identifiquem o inventário excessivo antes dele tornar-se obsoleto. Este artigo propõe um método para selecionar variáveis independentes em modelos de regressão preditivos com vistas à projeção do inventário ao longo do tempo, com o objetivo de reduzir o risco de obsolescência futuro. A abordagem proposta possui cinco passos, sendo os quatro primeiros dedicados à identificação dos fatores que contribuem para a obsolescência do inventário, a classificação do estoque em categorias e faixas de idade, a seleção de variáveis em contexto de PLS, a modelagem de regressão para projeção da idade do inventário ao longo do tempo e a definição de diretrizes para redução do risco de obsolescência. O quinto passo do método utiliza o conceito do ciclo PDCA buscando a melhoria contínua do processo e dos resultados. Na aplicação em uma indústria de bens de consumo, o método previu com precisão superior a $95 \%$ o montante do inventário por faixa de idade em um horizonte de seis meses.

PALAVRAS-CHAVE: Gestão de Estoques. Obsolescência. Seleção de Variáveis. PLS. Regressão Múltipla. 


\section{INTRODUÇÃO}

Algumas empresas destacam-se pelas vantagens competitivas conquistadas através de suas estratégias de gestão, e cuja manutenção depende da capacidade de gerenciamento financeiro e da habilidade da empresa em avaliar uma grande quantidade de informações. A utilização de ferramental quantitativo, como a modelagem de regressão, oferece subsídios importantes para tomada de decisão (Montgomery, 2008; Kasznar e Gonçalves, 2011).

A má gestão do capital de giro reduz a liquidez e limita a obtenção de vantagens competitivas, enquanto a má gestão do estoque compromete a rentabilidade e a capacidade de sobrevivência a longo prazo. A incerteza do mercado, variações da demanda, inovações tecnológicas e ciclo de vida curto dos produtos aumentam o risco de falta, excesso e obsolescência do inventário, tornando a definição de medidas de controle da obsolescência um importante instrumento de gestão (Porter, 1992; Beamon, 1999; Fisher, 2003; Wild, 2007; Mundstock, 2008, apud Motta, 1996; Panigrahi, 2013).

A literatura indica que a maioria dos modelos matemáticos de gestão de inventário envolve questões relativas à classificação de inventários, métodos de previsão, excesso ou falta de inventário, gestão de componentes de produtos com longo ciclo de vida, gestão de peças de reposição e processos de suprimentos. Poucas pesquisas nesta área abordam os processos de envelhecimento do inventário e o risco de obsolescência, e quando o fazem, não projetam o risco de obsolescência futuro nem visam à criação de estratégias para redução do risco de obsolescência (Wanke, 2005; Wong et al., 2006; Boylan et al., 2008; Nenes et al., 2010; Syntetos et al., 2010; Bonney e Jaber, 2011; Pinçe e Dekker, 2011; Bakker et al., 2012; Wu, 2013).

Este artigo propõe uma abordagem para selecionar variáveis independentes em modelos de regressão preditivos com vistas à projeção do inventário ao longo do tempo, reduzindo o risco de obsolescência futuro através da (i) investigação dos fatores que contribuem para o aumento da idade do inventário; (ii) classificação do estoque e dos produtos em faixas de idade; (iii) definição do conjunto de variáveis mais apropriado para projeção do inventário ao longo do tempo; e (iv) definição do conjunto de diretrizes mais apropriadas para redução do inventário com risco de obsolescência através de um processo de melhoria contínua.

\section{FUNDAMENTAÇÃO TEÓRICA}

\section{Diretrizes para Redução do Inventário com Risco de Obsolescência}

Panigrahi (2013) define inventário como o estoque dos produtos que uma empresa oferece para venda e os vários componentes que compõem esses produtos. Para Chopra e Sodhi (2004), o risco de inventário depende do valor do produto, da taxa de obsolescência e da incerteza da demanda e da oferta.

Obsolescência é a diminuição do valor, utilidade ou produtividade de um produto ou de seus componentes, devido a diminuição da conveniência e/ou função decorrente de novas invenções, mudanças no design, desenvolvimento 
tecnológico, melhoria do processo de produção, mudança no uso ou nas demandas dos usuários, incompatibilidade do ciclo de vida entre o produto acabado e seus componentes, mudanças climáticas ou alterações políticas ou de legislação (Kumar e Saranga, 2010; Tang e Musa, 2011; Pourakbar et al., 2012; Bakker et al., 2012; Krajčovič e Plinta, 2012; Butt et al, 2015; Pingle, 2015; Kessler e Brendel, 2016).

A obsolescência de estoques tornou-se um fenômeno de destaque nas organizações, demandando a implementação de medidas e métodos que identifiquem o inventário excessivo antes dele tornar-se obsoleto (Thummalapalli, 2010; Krajčovič e Plinta, 2012). Singh et al. (2008) afirmam que muitas empresas operam com sistemas de previsão e planejamento deficitários e não confiáveis, podendo levar ao excesso de estoque obsoleto. Van Donk e Van Der Vaart (2005) alertam que a ausência de informações resulta em custos desnecessários de inventário e risco de obsolescência. Syntetos et al. (2010) citam a importância de considerar a obsolescência do inventário em modelos previsão e controle de estoques. Chae (2009) aponta que estoques obsoletos são a principal fonte de aumento do custo total do inventário.

Para Jennings (2015), muitas abordagens para previsão de obsolescência baseiam-se em entradas manuais e melhores estimativas de especialistas da área. A pesquisa de Van Jaarsveld e Dekker (2010) afirma que há poucas abordagens na literatura que incorporam o risco de obsolescência em modelos de gestão de estoque, e cita os trabalhos de Song e Zipking (1993 e 1996) sobre os efeitos da obsolescência na política de inventário em ambientes de demanda flutuante através de um modelo computacional. Beamon (1998) apresentou uma revisão da literatura sobre modelagem da cadeia de abastecimento multiestágio, na qual apenas o trabalho conduzido por Ichii et al. (1998) abordou a redução do estoque obsoleto através de um modelo para determinar o nível econômico de estoque. Teunter et al. (2011) avaliaram a literatura sobre demanda intermitente e risco de obsolescência, propondo um método de previsão da demanda com foco na redução do risco de obsolescência. Kholopane (2016) apresenta uma aplicação da ferramenta Seis Sigma integrada com os sistemas de gestão de inventário para melhorar o controle de estoque e minimizar o risco de obsolescência. Pingle (2015) propõe um método para gestão da obsolescência de inventário através da Teoria da Utilidade Multiatributo (MAUT). Yang e Williams (2009) propõem uma metodologia para caracterizar as tendências futuras na geração de computadores obsoletos a partir de dados históricos de vendas. Zhang et al. (2011) apresentam um modelo para estimar o inventário futuro de eletrodomésticos para efeitos de logística reversa. Sandborn et al. (2011) propõem uma metodologia para geração de algoritmos de previsão das datas de obsolescência de componentes eletrônicos com base no tempo de vida e dados de eventos de obsolescência anteriores. Kumar e Saranga (2010) revisaram a literatura sobre o risco de obsolescência de componentes e propuseram um método para definir o tempo ótimo para o redesenho dos produtos e redução do risco de obsolescência de seus componentes. Nenes et al. (2010) incluem o risco de obsolescência por excesso de inventário para evitar baixos níveis de atendimento aos clientes em um sistema informatizado capaz de identificar porém não prever - itens obsoletos. Battini et al. (2014) consideram o risco de obsolescência do inventário no modelo proposto para calcular os custos totais de abastecimento. Pinçe e Dekker (2011) propõem um sistema de revisão contínua do inventário incorporando o risco de obsolescência em situações de queda da 
demanda em um momento futuro. Bartels et al. (2012) propõem a utilização do ciclo PDCA para melhoria contínua do plano de gerenciamento de obsolescência. Bakker et al. (2012) e Janssen et al. (2016) tratam da gestão de estoques de itens perecíveis abordando os processos de envelhecimento do inventário. Wu (2013) propõe um modelo de previsão multiperíodo de baixa contábil do inventário incluindo a idade do inventário através de procedimentos heurísticos de tempo polinomial sequencial. A pesquisa de Chen et al. (2011) com semicondutores mostra que as características desta indústria resultam em altos riscos de obsolescência tecnológica. Li et al. (2014) propõe um sistema inteligente de gerenciamento que permite o monitoramento e analise do envelhecimento do inventário para reduzir o excesso de estoque. Westerman (2015) propõe um indicador da idade do inventário para maximizar o capital de giro. Raza et al. (2017) citam o impacto negativo do envelhecimento do inventário em um estudo de caso na indústria automotiva. Meng et al. (2014) apresentaram um modelo matemático proativo de gerenciamento de obsolescência para sistemas de longa vida útil.

Kaufmann e Gaeckler (2015) conduziram um estudo sobre o uso de PLS (Partial Least Squares) em 75 artigos publicados de 2002 a 2013, indicando seu potencial e restrições de uso nos processos de gestão da cadeia de suprimentos. Dentre os artigos pesquisados, nenhum dos estudos aborda a redução do risco de obsolescência, e apenas o trabalho de Claassen et al. (2008) apresenta uma aplicação de regressão PLS para planejamento do inventário.

\section{FERRAMENTAS MULTIVARIADAS}

Os métodos estatísticos dividem-se em univariados, bivariados e multivariados. Os métodos multivariados aplicam-se a conjuntos de dados que incluem medições simultâneas de diversas variáveis, com o propósito de medir, explicar e prever o grau de relacionamento entre as variáveis. A maioria das análises estatísticas é multivariada por natureza, e a dificuldade em realizar pesquisas complexas com análises univariadas contribui para que os métodos multivariados sejam amplamente utilizados (Vicini e Souza; 2005; Mingoti, 2005; Tabachnick e Fidell, 2007; Johnson e Wichern, 2007; Bakke et al., 2008; Hair et al., 2009; Liland, 2011).

A correta aplicação das técnicas multivariadas demanda o entendimento dos conceitos de classificação de variáveis (quanto ao tipo: dependentes ou independentes, e; quanto a natureza: quantitativas ou qualitativas), escala de medida (métricas ou não métricas), variate, e medição multivariada (Tabachnick e Fidel, 2007; Melo e Hepp, 2008; Hair et al., 2009; Schielzeth, 2010; Agresti e Kateri, 2011).

O conceito de variate refere-se a uma nova variável gerada através da combinação linear das variáveis originais com pesos determinados empiricamente. Enquanto as variáveis são definidas pelo pesquisador, os pesos são determinados pela técnica multivariada, resultando em uma combinação de todo o conjunto de variáveis que melhor atinja o objetivo da análise. Uma variate de $n$ variáveis ponderadas pode ser descrita matematicamente conforme a equação (1), na qual $x_{n}$ é a variável observada e $w_{n}$ é o peso determinado pela técnica multivariada. 


$$
\text { variate }=w_{1} x_{1}+w_{2} x_{2}+w_{3} x_{3}+\cdots+w_{n} x_{n}
$$

A medição multivariada (reunião de diversas variáveis em uma medida composta para representar um conceito) busca a redução dos erros de medição através da validação dos dados e do aumento da confiabilidade das variáveis individuais (Hair et al., 2009).

Entre os pré-requisitos para utilização das ferramentas multivariadas estão a distribuição normal multivariada e a natureza aleatória e inter-relacionada das variáveis (Vicini e Souza, 2005; Ayres et. al., 2007; Johnson e Wichern, 2007; Tabachnick e Fidell, 2007; Hair et al., 2009).

Hair et al. (2009) propõem uma classificação das técnicas multivariadas em função do objetivo de pesquisa, conforme exposto no Quadro 1.

Quadro 1 - Classificação das técnicas multivariadas

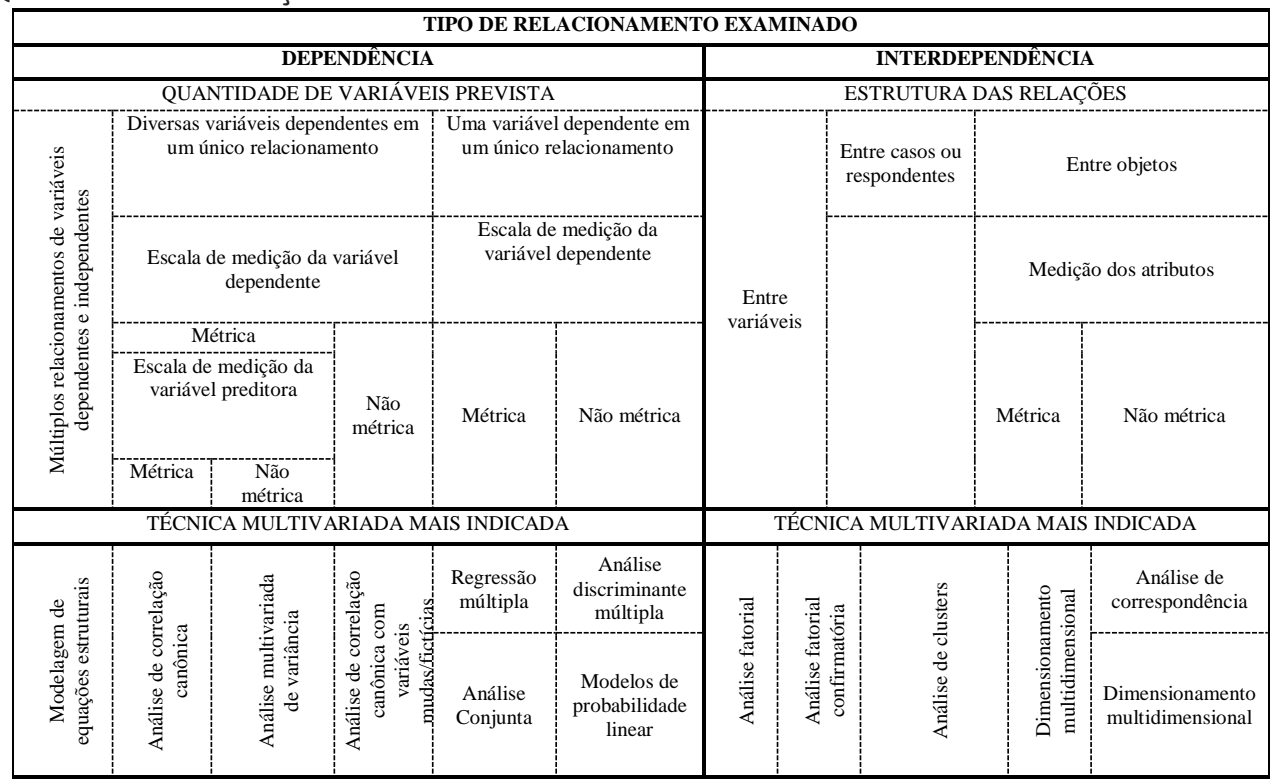

Fonte: Adaptado de Hair et al. (2009)

Dado o objeto de estudo deste trabalho, as técnicas de regressão linear múltipla e regressão PLS serão abordadas nos próximos tópicos.

\section{Regressão Linear Múltipla}

O problema de regressão (modelar uma ou mais variáveis dependentes y por meio de um conjunto de variáveis independentes $x$ ) é um dos problemas de dados analíticos mais comuns em ciência e tecnologia. Tradicionalmente, a modelagem de $y$ por meio de $x$ é feita usando métodos de regressão linear simples, regressão linear múltipla e regressão linear múltipla multivariada (Wold et al., 2001; Rencher, 2003; James et al., 2013).

$\mathrm{Na}$ regressão linear múltipla procura-se minimizar a soma do desvio quadrado entre a resposta ajustada e a resposta verdadeira no espaço medido pelas variáveis explicativas. Neste processo, cada variável independente é ponderada para assegurar a máxima previsão a partir do conjunto de variáveis independentes. Os pesos utilizados no processo de ponderação indicam a contribuição relativa de cada variável independente para a previsão. A aplicação da técnica demanda que os dados sejam métricos (ou tenham sido 
transformados através da codificação de variáveis fictícias) e que haja a separação das variáveis dependentes e independentes (Kettaneh et al., 2005; Tabachnick e Fidell, 2007; Hair et al., 2009; Liland, 2011).

Para aumentar o poder de predição do modelo deve-se procurar variáveis independentes com baixa colinearidade ou multicolinearidade com as demais variáveis independentes, mas com alta correlação com a variável dependente. Como regra, o conjunto ideal de variáveis independentes é o menor conjunto confiável, não correlacionado, que cobre as principais características da variável dependente (Tabachnick e Fidell, 2007; Hair et al., 2009). Desde que o número de observações seja maior do que o número de variáveis explicativas e que estas não sejam multicolineares, a regressão linear múltipla é estável (Liland, 2011).

O modelo de regressão linear com uma única resposta é descrito conforme a equação (2), onde $y^{\prime}$ é o valor previsto de $y$ (variável dependente), $b_{0}$ é o valor de $y^{\prime}$ quando todos os $x$ forem igual a zero, $b_{1}$ a $b_{k}$ representam os coeficientes de regressão e $x_{1}$ a $x_{k}$ são as variáveis independentes e $\varepsilon$ é o erro de predição (residual) (Rencher, 2003; Johnson e Wichern, 2007; Tabachnick e Fidell, 2007; Hair et al., 2009; James et al., 2013).

$$
y^{\prime}=\beta_{0}+\beta_{1} x_{1}+\beta_{2} x_{2}+\cdots+\beta_{k} x_{k}+\varepsilon
$$

Esta equação assume que nenhuma condição adicional é necessária para prever $y$, e que toda variação restante em y é puramente aleatória e imprevisível. Com $n$ observações independentes sobre $y$ e os valores associados de $x$, expressando cada $y$ em uma amostra de $n$ observações como uma função linear de $x$, o modelo completo apresenta-se conforme descrito na equação (3) (Rencher, 2003; Johnson e Wichern, 2007).

$$
\begin{gathered}
y_{1}^{\prime}=\beta_{0}+\beta_{1} x_{11}+\beta_{2} x_{12}+\cdots+\beta_{k} x_{1 k}+\varepsilon \\
y^{\prime}{ }_{2}=\beta_{0}+\beta_{1} x_{21}+\beta_{2} x_{22}+\cdots+\beta_{k} x_{2 k}+\varepsilon \\
\vdots \\
y_{n}^{\prime}=\beta_{0}+\beta_{1} x_{n 1}+\beta_{2} x_{n 2}+\cdots+\beta_{k} x_{n k}+\varepsilon
\end{gathered}
$$

Os coeficientes de regressão que melhor se ajustam ao modelo produzem uma equação de predição para a qual as diferenças quadradas entre $y$ e $y^{\prime}$ estão em seu valor mínimo $\left(\left[y-y^{\prime}\right]^{2}\right)$, o que é denominado solução de mínimos quadrados (least squares) (Tabachnick e Fidell, 2007; Hair et al., 2009).

A função estatística $F$ (F-statistic) avalia a relevância das variáveis independentes na predição das variáveis dependentes, sendo esperado um valor F maior do que 1 quando há relação entre a resposta e os preditores, e um valor F próximo a 1 quando não há relação entre a resposta e os preditores (Mário, 2002; James et al., 2013). O cálculo da função estatística $F$ é realizado conforme as equações (4), (5) e (6) onde y' é o valor previsto de $y$, TSS é a soma total dos quadrados (Total Sum of Squares - variância total na resposta $y$, ou quantidade de variabilidade inerente à resposta antes da regressão ser realizada), RSS é a soma total dos quadrados residuais (Residual Sum of Squares - diferença entre o valor da resposta observada e o valor da resposta prevista pelo modelo linear), $n$ é o número de observações e $p$ é o número de variáveis independentes (James et al., 2013; Mário, 2002).

$$
F=\frac{(T S S-R S S) / p}{R S S /(n-p-1)}
$$




$$
\begin{aligned}
& T S S=\sum\left(y_{i}-y^{\prime}\right)^{2} \\
& R S S=\sum\left(y_{i}-y_{i}^{\prime}\right)^{2}
\end{aligned}
$$

Os métodos mais comuns para mensurar a precisão da previsão são o RSE (Residual Standard Error) e o coeficiente de determinação $R^{2}$. O RSE é uma estimativa do desvio padrão do erro de predição descrito conforme a equação (7), onde $p$ é o número de variáveis independentes e RSS é a soma total dos quadrados residuais (James et al., 2013).

$$
R S E=\sqrt{\frac{1}{n-p-1} R S S}
$$

O RSE fornece uma medida absoluta da falta de ajuste do modelo aos dados expressa em unidades de $y$, logo, nem sempre é claro o que constitui um bom RSE. O coeficiente de determinação $R^{2}$ fornece uma medida de ajuste em forma de proporção da variância explicada, assumindo um valor entre 0 e 1 independente da escala de $y$. Um valor de $\mathrm{R}^{2}$ próximo de 1 indica que o modelo explica uma grande porção da variância na variável de resposta (James et al., 2013). Como $R^{2}$ é unitário e adimensional, seu conceito é intuitivo e extremamente útil como índice sumário para modelos estatísticos ou comparativo entre diferentes estudos (Nakagawa e Schielzeth, 2013).

Tabachnick e Fidell (2007) e Hair et al. (2009) apresentam de forma detalhada os conceitos envolvidos no processo de mínimos quadrados. Antes de estimar a regressão define-se a linha de base sobre a qual será comparada a habilidade de predição do modelo (simples média das variáveis independentes). Como a média não irá prever perfeitamente cada valor das variáveis independentes, e como a observação dos erros na predição das variáveis dependentes através da média das variáveis independentes soma zero, deve-se elevar cada erro ao quadrado e somar os resultados obtidos. A soma dos quadrados de regressão é a soma das diferenças dos quadrados entre o previsto em $y^{\prime}$ e a média de $y$, enquanto a soma de quadrados residuais é a soma das diferenças dos quadrados entre os valores observados de $y$ e as previsões do modelo $y^{\prime}$, representando os erros na previsão. A correlação múltipla é a correlação entre os valores previstos e os valores obtidos de $y$, e a correlação múltipla ao quadrado $\left(R^{2}\right)$ é a proporção da soma dos quadrados de regressão na soma total dos quadrados para y. $O R^{2}$ também pode ser observado nas correlações entre cada uma das variáveis independentes e a variável dependente.

O coeficiente de determinação $R^{2}$ é representado pela equação (8), sendo que $S S_{r}$ representa a soma dos quadrados da diferença entre a média da variável dependente e os valores estimados para todas as observações, $S S_{e}$ representa a soma dos quadrados dos resíduos para todas as observações e $S Y_{y}$ representa a soma dos quadrados totais de y (Ferrer, et al., 2008; Masiero e Anzanello, 2011; Nakagawa e Schielzeth, 2013; James et al., 2013).

$$
R^{2}=\frac{S S_{y}}{S Y_{y}}=1-\frac{S S_{e}}{S Y_{y}}
$$

O coeficiente de determinação $R^{2}$ deve apresentar propriedades específicas para que sejam utilizados em modelos de regressão, as quais estão relacionadas no Quadro 2. 
Quadro 2 - Propriedades do $\mathrm{R}^{2}$ para modelos de regressão

\begin{tabular}{|c|l|}
\hline \multicolumn{2}{|c|}{ PROPRIEDADES DO R ${ }^{2}$ PARA MODELOS DE REGRESSÃO } \\
\hline 1 & $\mathrm{R}^{2}$ deve ter utilidade como medida adequação geral do modelo e deve ser de fácil interpretação \\
\hline 2 & $\mathrm{R}^{2}$ deve ser adimensional \\
\hline 3 & $\begin{array}{l}\mathrm{R}^{2} \text { deve variar de 0 a 1, sendo zero a ausência total de ajuste e 1 uma adequação perfeita do ajuste ao } \\
\text { modelo }\end{array}$ \\
\hline 4 & $\mathrm{R}^{2}$ deve ser suficientemente geral para ser aplicável a qualquer tipo de modelo \\
\hline 5 & Os valores de $\mathrm{R}^{2}$ não devem ser afetados por diferentes técnicas de ajuste do modelo \\
\hline 6 & $\begin{array}{l}\text { Os valores de } \mathrm{R}^{2} \text { para diferentes modelos ajustados ao mesmo conjunto de dados devem ser diretamente } \\
\text { comparáveis }\end{array}$ \\
\hline 7 & Os valores relativos de $\mathrm{R}^{2}$ devem ser comparáveis aos de outros métodos válidos de ajuste da regressão \\
\hline 8 & Todos os resíduos (positivos e negativos) devem ser ponderados igualmente pelo $\mathrm{R}^{2}$ \\
\hline 9 & O valor de $\mathrm{R}^{2}$ não deve diminuir à medida em que novas variáveis independentes são adicionadas \\
\hline 10 & $\begin{array}{l}\text { O valor de } \mathrm{R}^{2} \text { baseado na soma residual de quadrados deve coincidir com o valor de } \mathrm{R}^{2} \text { baseado na } \\
\text { soma de quadrados explicada }\end{array}$ \\
\hline 11 & Os valores de $\mathrm{R}^{2}$ e a significância estatística dos parâmetros de declive devem mostrar correspondência \\
\hline 12 & O valor de $\mathrm{R}^{2}$ deve ser interpretável em termos do conteúdo de informação dos dados \\
\hline
\end{tabular}

Fonte: Adaptado de Adaptado de Orelien e Edwards (2008) e Nakagawa e Schielzeth (2013)

$O \mathrm{R}^{2}$ ajustado considera o número de variáveis independentes incluídas na equação de regressão e o tamanho da amostra. Embora o $R^{2}$ sempre aumente ao adicionar variáveis ao modelo, o $\mathrm{R}^{2}$ ajustado pode cair se as variáveis acrescentadas tiverem pouco poder explicativo ou se os graus de liberdade se tornarem demasiadamente pequenos (Hair et al., 2009). O $R^{2}$ ajustado apresentase conforme a equação (9), onde RSS é a soma total dos quadrados residuais (Residual Sum of Squares), TSS é a soma total dos quadrados (Total Sum of Squares), $n$ é o número de observações e $p$ é o número de variáveis independentes (James et al., 2013).

$$
R^{2} \text { ajustado }=\frac{R S S /(n-p-1)}{T S S /(n-1)}
$$

Para James et al. (2013), a decisão entre utilizar o coeficiente $R^{2}$ ou outros métodos de ajuste do modelo de previsão depende da precisão e da interpretabilidade do modelo desejada pelo pesquisador. $O \mathrm{R}^{2}$ leva a uma alta precisão de resposta quando a relação entre a resposta e os preditores for aproximadamente linear e o número de observações $(p)$ for muito maior que o número de variáveis $(n)$, e a remoção das variáveis que não estejam associadas à resposta conduz a um modelo de melhor interpretabilidade.

\section{Regressão PLS}

O método Partial Least Squares (PLS) é uma generalização da regressão linear múltipla capaz de analisar dados altamente colineares (correlacionados), ruidosos e numerosos, além de modelar simultaneamente várias variáveis de resposta (Wold et al., 2001; Höskuldsson, 2001; Zimmer e Anzanello, 2014). O PLS atende a uma ampla classe de métodos (algoritmos de mínimos quadrados) para modelagem das relações entre conjuntos de dados observados por meio de variáveis latentes (Rosipal e Kramer, 2006; Henseler et al., 2009).

A regressão $P L S$ é um método baseado em projeção que relaciona a matriz $X$ (variáveis de previsão) à matriz Y (variáveis dependentes), gerando um conjunto de parâmetros que fornecem informações sobre a estrutura e o comportamento de $X$ e de $Y$, produzindo estimativas estáveis dado que a correlação entre as variáveis é considerada na construção do modelo. O algoritmo PLS é uma 
sequência de regressões em termos de vetores de peso, e seu algoritmo básico inclui: (i) estimativa iterativa das pontuações das variáveis latentes; (ii) estimativa de pesos/cargas exteriores e coeficientes de caminho, e; (iii) avaliação dos parâmetros de localização (Wold et al., 2001; Miletic et al., 2004; AlGhazzawi e Lennox, 2009; Henseler et al., 2009).

Os fundamentos matemáticos da regressão PLS são detalhados por Wold et al. (2001), AlGhazzawi e Lennox (2009), Zimmer (2012) e Anzanello (2013), como segue: considere uma matriz $\mathrm{X}$, de dimensão $\mathrm{K} \times \mathrm{N}$, e uma matriz $\mathrm{Y}$, de dimensão $\mathrm{M} \times \mathrm{N}$ (sendo $\mathrm{K}$ o número de variáveis de processo, $\mathrm{M}$ o número de variáveis de resposta e $\mathrm{N}$ o número de observações); o vetor xi $\left(x_{i 1}, x_{i 2}, \ldots, x_{i k}\right)$ representa a observação $i$ para cada variável de processo $k$, enquanto o vetor $y_{i}\left(y_{i 1}, y_{i 2}, \ldots, y_{i m}\right)$ representa a observação $i$ para cada variável de resposta $m$; a regressão PLS gera a variáveis latentes (combinações lineares) $t_{a}(a=1,2, \ldots, a)$ a partir das variáveis originais; as combinações são ortogonais entre si e usadas com propósitos de predição e controle de processo; a definição do número de componentes a serem mantidos no modelo se dá através da avaliação da significância de cada componente em termos de predição, interrompendo-se a inclusão de componentes no modelo quando os mesmos deixam de ser significativos. As variáveis latentes $t_{a}$ são combinações lineares independentes das variáveis $x$ com coeficientes $w_{a}\left(w_{1 a}, w_{2 a}, \ldots, w_{k a}\right)$, conforme a equação (10):

$$
t_{i a}=w_{1 a}+w_{1 x}+w_{2 a}+w_{2 x}+\cdots+w_{k a}+w_{k x}=w^{\prime}{ }_{a} x_{i}
$$

$O$ vetor $w_{\text {a }}$ representa o peso da variável de processo $k$ no componente $a$, sendo importante ressaltar que também leva em conta a influência das variáveis de produto. As variáveis latentes $u_{\mathrm{a}}(a=1,2, \ldots, a)$ são combinações lineares das variáveis $y .0$ vetor $c_{a}\left(c_{1 a}, c_{2 a}, \ldots, c_{m a}\right)$ representa o peso de cada variável de produto $m$ no componente $a$, conforme a equação (11):

$$
u_{i a}=c_{1 a}+y_{1 x}+c_{2 a}+y_{2 x}+\cdots+c_{k a}+y_{k x}=c^{\prime}{ }_{a} y_{i}
$$

Os vetores $w_{\mathrm{a}}$ e $c_{\mathrm{a}}$ são escolhidos de forma a maximizar a covariância entre os componentes $t_{\mathrm{a}}$ e $u_{\mathrm{a}}$ (concentram informações sobre as observações e suas semelhanças em relação ao modelo), e fornecem informações sobre como as variáveis se combinam para formar a relação quantitativa entre $X$ e $Y$, apontando as variáveis $x$ de maior relevância (maiores valores de $w_{\mathrm{a}}$ ). Multiplicando o vetor de cargas das variáveis de processo $p_{\mathrm{a}}\left(p_{1 \mathrm{a}}, p_{2 \mathrm{a}}, \ldots, p_{\mathrm{ka}}\right)$, pelo vetor $t_{\mathrm{a}}$, pode-se reconstituir a matriz $X$ com valores reduzidos dos resíduos $e_{\mathrm{ik}}$, conforme a equação (12), cuja representação matricial é $x=t p^{\prime}+e$.

$$
x_{i k}=\sum_{a} t_{i a} p_{a k}+e_{i k}
$$

A predição das variáveis de resposta y pode ser obtida pela multiplicação de $u_{a}$ pelos coeficientes $c_{a}$, conforme a equação (13), cuja representação matricial é $y=u c^{\prime}+g$.

$$
y_{i m}=\sum_{a} u_{i a} c_{a m}+g_{i m}
$$

A equação (14) apresenta os coeficientes da regressão PLS, cuja representação matricial é $b=w^{*} c^{\prime}$. Os resíduos da predição são representados por $w^{*}{ }_{k a}=w_{\text {ka }}\left(p_{\text {ka }} w_{\text {ka }}\right)-1$.

$$
b_{m k}=\sum_{a} c_{m a} w_{k a}^{*}+f_{i m}
$$


Substituindo-se as equações anteriores chega-se ao formato tradicional para o modelo de regressão proposto por Wold et al. (2001), conforme a equação (15), cuja representação matricial é $y=x b+f$.

$$
y_{i m}=\sum_{a} b_{m k} x_{i k}+f_{i m}
$$

Com o grande número de variáveis coletadas nos processos industriais, é comum que as variáveis estejam localizadas em diferentes intervalos numéricos, influenciando sua variância. Como os métodos PLS são dependentes da variância, é geralmente necessário colocar os dados em uma mesma escala para torná-los mais adequados para a análise (Ferrer, et al., 2008).

\section{SELEÇÃO DE VARIÁVEIS}

Um modelo estatisticamente válido pode ser obtido utilizando todas as variáveis de determinado conjunto de dados. Em grandes conjuntos de dados, porém, é comum observar-se muitas variáveis irrelevantes, ruidosas ou não confiáveis, fazendo-se necessária a sua remoção para melhorar as previsões, reduzir a complexidade, melhorar as propriedades estatísticas, reduzir os requisitos de medição e armazenamento, reduzir os tempos de formatação e utilização dos dados e definir preditores mais rápidos e rentáveis (Guyon e Elisseeff, 2003; Balcaen e Ooghe, 2006; Andersen e Bro, 2010). A redução do número de variáveis, reduzindo a dimensão de tal forma que os aspectos mais significativos dos dados sejam representados em um subconjunto de dados úteis a partir do conjunto de dados de entrada, é o objetivo dos métodos de seleção de variáveis (Blum e Langley, 1997; Dash e Liu, 2003, apud Devijver e Kittler, 1982; Tabakhi et al., 2014).

Para Dash e Liu (2003), seleção de variáveis é uma técnica eficaz para classificação de dados e redução de dimensionalidade, podendo ser usada para encontrar um subconjunto ótimo de características relevantes no qual a precisão global de classificação é aumentada enquanto a dimensão dos dados é reduzida. Para Gnana et al. (2016), seleção de variáveis é um processo de remoção das características irrelevantes e redundantes de um conjunto de dados, a fim de melhorar o desempenho dos algoritmos em termos de precisão e tempo para construção do modelo. Para Brosofske et al. (2014) modelos de seleção de variáveis incluem variáveis independentes que estão estatisticamente relacionadas com a resposta e descartam aquelas que são irrelevantes ou redundantes. O conceito de relevância para seleção de variáveis é discutido detalhadamente na pesquisa realizada por Blum e Langley (1997).

Embora a identificação e seleção de variáveis possa ser feita de forma empírica (através do conhecimento de especialistas, popularidade na literatura e sucesso preditivo em pesquisas anteriores), esta abordagem é frequentemente sujeita a equívocos, fazendo com que, ao final da seleção manual, o pesquisador não tenha certeza de que a previsão é a melhor possível (Gauchi e Chagnon, 2001; Balcaen e Ooghe, 2006; Masiero e Anzanello, 2011).

Os métodos de seleção de variáveis tornaram-se objeto de pesquisa em diversas áreas de aplicação e mineração de dados nas quais conjuntos de dados com de grande quantidade de variáveis estão disponíveis para análise (Guyon e Elisseeff, 2003; Liu e Yu, 2005; Stein et al., 2014; Cervo e Anzanello, 2015). Os recentes avanços da tecnologia aumentam a facilidade e reduzem o custo de 
medição de múltiplas variáveis por objeto, tornando a seleção de variáveis cada vez mais importante para a redução e interpretação de dados (Mehmood et al., 2012). A vasta literatura sobre seleção de variáveis apresenta a sua utilização nas mais diversas aplicações (Yu e Liu, 2004; Balcaen e Ooghe, 2006; Saeys et al., 2007; Anzanello et al., 2009; Masiero e Anzanello, 2011; Zimmer, 2012; Anzanello, 2013; Stein et al., 2014; Cervo e Anzanello, 2015).

\section{Seleção de Variáveis em Contexto de PLS}

Além da capacidade de previsão, os métodos PLS são apreciados pela capacidade de redução da quantidade de variáveis do modelo, especialmente em ambientes com grande quantidade de dados (Ferrer, et al., 2008). A regressão PLS (PLSR) é uma técnica PLS para a redução da dimensão, usada para reduzir o número de variáveis explanatórias num problema de regressão, eliminar a multicolinearidade do conjunto de variáveis explanatórias e estimar o subconjunto de variáveis explicativas ideais para prever as variáveis dependentes (Roy e Roy, 2008; Mateos-Aparicio, 2011).

Entre as abordagens estatísticas clássicas para seleção de variáveis destacam-se a Forward Selection (FS - escolha das variáveis que dão os melhores prognósticos, uma a uma, a partir da variável com menor erro de predição), a Backward Selection (BS - assemelha-se à FS, porém usa o modelo completo a partir do qual as variáveis que contribuem menos para as previsões são removidos uma a uma), Stepwise Multiple Regression (SMR - combinação de FS e $\mathrm{BS}$, seleciona variáveis como na $\mathrm{FS}$, removendo variáveis escolhidas caso alguma variável adicionada posteriormente torne-a menos importante), Simple Regression (SR - começa pela regressão simples para cada uma das variáveis explicativas, mantendo apenas as variáveis que obedecem a determinados limites) e a Best Subset Selection (BSS - analisa modelos de regressão linear múltipla em todas as combinações de variáveis e escolhe a combinação que confere o melhor ajuste ao modelo) (Gauchi e Chagnon, 2001, Chong e Jun, 2005; Andersen e Bro, 2010).

A seleção de variáveis a partir de FS, BS, SMR ou BSS resulta na criação de diferentes modelos contendo um determinado subconjunto de variáveis independentes. Como regra, o modelo contendo todos os preditores terá sempre o menor RSS e o maior $\mathrm{R}^{2}$, porém, estes indicadores não são adequados para escolha do modelo com menor erro de predição entre modelos com diferentes números de preditores. Entre as técnicas adequadas para selecionar o melhor modelo com relação ao erro de teste estão a $\mathrm{Cp}$ (critério $\mathrm{Cp}$ de Mallow), AIC (Akaike Information Criterion), BIC (Bayesian Information Criterion) e o $\mathrm{R}^{2}$ ajustado (James et al., 2013; Johnson e Wichern, 2007).

A literatura apresenta diferentes classificações das técnicas de seleção de variáveis com base em PLSR. O Quadro 3 traz as principais características de cada método, suas vantagens e desvantagens. 
Quadro 3-Técnicas de seleção de variáveis

\begin{tabular}{|l|l|}
\hline & \multicolumn{1}{|c}{ FILTRO (FILTER) } \\
\hline Métodos de filtro são simples e fornecem um ranking das variáveis com \\
relação a alguma medida de importância. Identificam as variáveis \\
relevantes dentro de um conjunto de dados e selecionam as variáveis como \\
um passo de pré-processamento, independentemente do preditor escolhido. \\
Avaliam a relevância das variáveis olhando somente para as propriedades \\
intrínsecas do conjunto de dados. Na maioria dos casos, a relevância das \\
variáveis é calculada através de coeficientes de correlação removendo e as \\
variáveis de baixa relevância.
\end{tabular}
ENVELOPE (WRAPPER)

Método simples e eficaz de seleção de variáveis que utiliza o método de filtro de forma iterativa, identificando as variáveis que serão reintroduzidas no modelo de seleção de variáveis como um subconjunto de dados, ocorrendo o "envelopamento" dos dados para produzir modelos reduzidos. Os subconjuntos de dados são escolhidos de acordo com o seu poder preditivo. A utilidade relativa dos subconjuntos de dados é avaliada, realizando uma busca no conjunto de dados por possíveis subconjuntos de dados, e vários subconjuntos de dados são gerados e avaliados.

\begin{tabular}{|c|c|}
\hline Vantagens & Desvantagens \\
\hline $\begin{array}{l}\text { - Simples; } \\
\text { - Interage com o classificador; } \\
\text { - Modela a dependência dos recursos. }\end{array}$ & $\begin{array}{l}\text { - Risco de sobre ajuste; } \\
\text { - Propenso a ficar preso em locais } \\
\text { ótimos (greedy search); } \\
\text { - Seleções dependentes do } \\
\text { classificador; } \\
\text { - Computacionalmente intensivo } \\
\text { (risco de NP-hard). }\end{array}$ \\
\hline
\end{tabular}
INCORPORADO (EMBEDDED)

O método incorporado orienta sua busca estimando mudanças no valor da função objetivo fazendo movimentos no espaço do subconjunto de dados. A busca de um subconjunto ótimo de recursos está embutida na construção do classificador, e pode ser visto como uma busca no espaço combinado de conjunto e subconjuntos de dados. As abordagens incorporadas são específicas para um determinado algoritmo de aprendizagem.

\begin{tabular}{l|l}
\hline \multicolumn{1}{|c}{ Vantagens } & \multicolumn{1}{|c}{ Desvantagens } \\
\hline - Melhor complexidade & $\begin{array}{l}\text { Seleções dependentes do } \\
\text { classificador. } \\
\text { computacional do que os métodos } \\
\text { envelopados; }\end{array}$ \\
- Modela a dependência dos recursos; & \\
- Melhor utilização dos dados & \\
disponíveis do que os métodos & \\
envelopados; & \\
- Atingem uma solução mais rápida & \\
do que os métodos envelopados. &
\end{tabular}

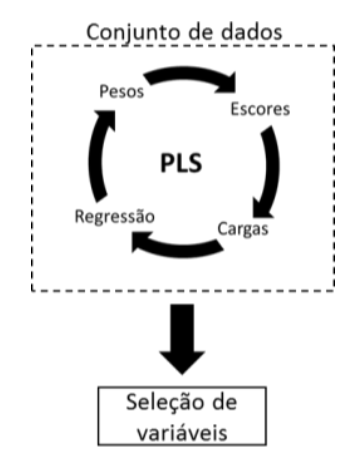

HÍBRIDO (HYBRID)

As abordagens híbridas selecionam as variáveis em duas fases: (i) reduzem o conjunto de dados original usando a abordagem de filtro; (ii) abordagem de envelope é aplicada para selecionar o melhor subconjunto de variáveis no conjunto de dados reduzido.

\begin{tabular}{|c|c|}
\hline Vantagens & Desvantagens \\
\hline $\begin{array}{l}\text { - Combina as vantagens dos métodos } \\
\text { filtro e envelope; } \\
\text { - Reduz a complexidade } \\
\text { computacional; } \\
\text { - Aplicação mais restrita em } \\
\text { comparação com os métodos de } \\
\text { filtragem, visto que usam o algoritmo } \\
\text { de aprendizado supervisionado no } \\
\text { processo de seleção de recurso; } \\
\text { - Menor risco de eliminar variáveis } \\
\text { relevantes do que na abordagem } \\
\text { filtro; } \\
\text { - Melhor processamento de conjunto } \\
\text { de dados de grande porte do que os } \\
\text { métodos de envelope. }\end{array}$ & $\begin{array}{l}\text { - Demandam mais tempo } \\
\text { computacional do que os métodos } \\
\text { de filtro. }\end{array}$ \\
\hline
\end{tabular}

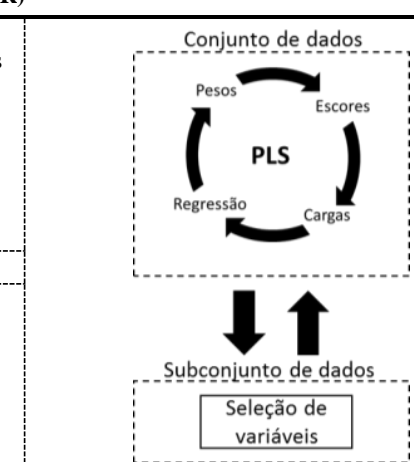




\section{MÉTODO}

A abordagem proposta para selecionar variáveis independentes em modelos de regressão preditivos com vistas à projeção do inventário ao longo do tempo, visando reduzir o risco de obsolescência futuro, contempla cinco etapas conforme exposto na Figura 1.

Figura 1 - Etapas do modelo proposto

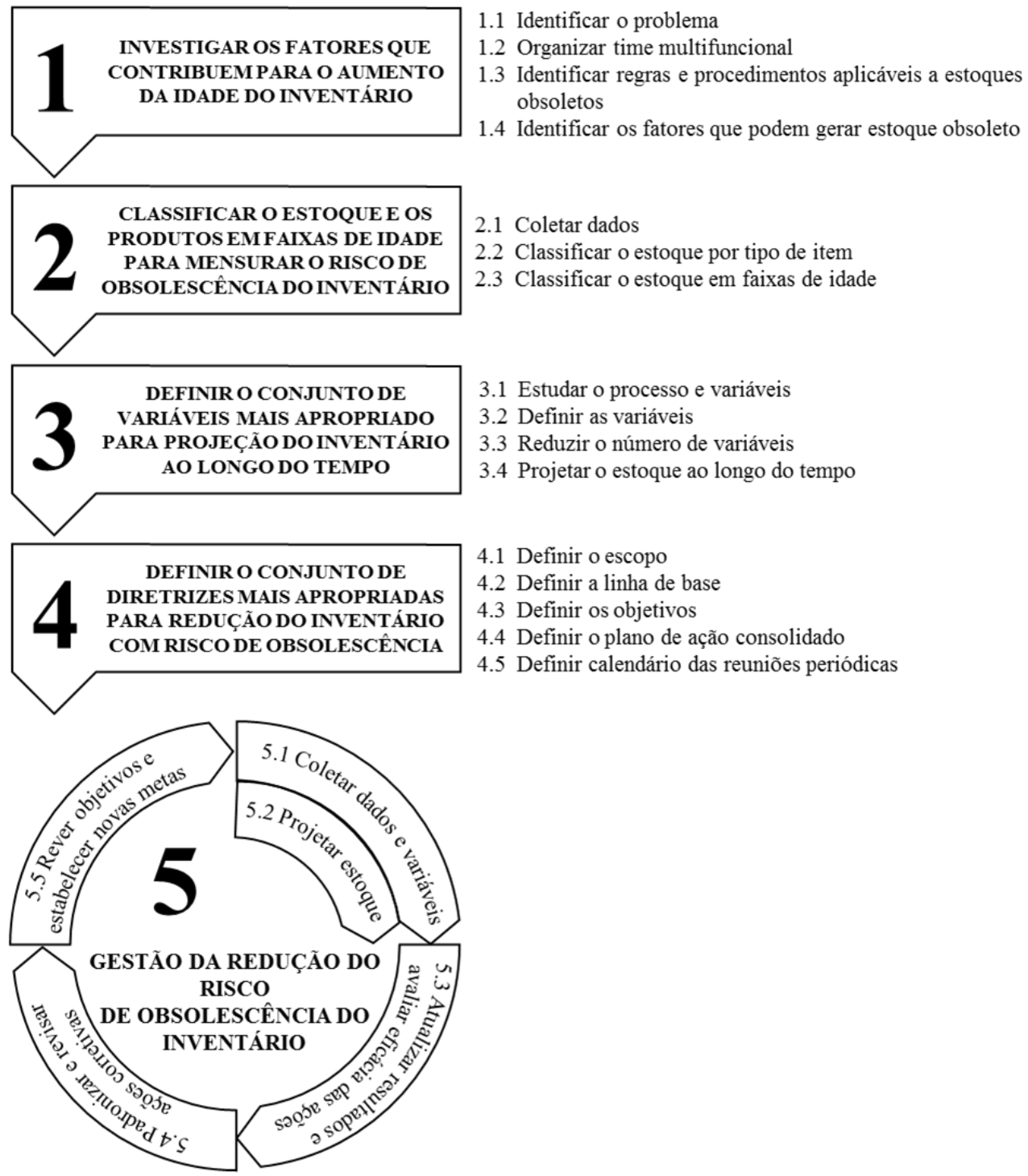

Fonte: Autoria própria (2017)

As primeiras quatro etapas do método compreendem a coleta e análise dos dados e do ambiente, a classificação do estoque, a criação de um cenário futuro com base na projeção do estoque ao longo do tempo e a proposição de ações corretivas para reduzir o risco de obsolescência futuro. Estas quatro etapas são realizadas uma única vez no início do projeto, e são fundamentais para que a melhoria contínua do processo seja perseguida através da etapa 5.

A etapa 5 deve ser realizada mensalmente de forma sistêmica, e consiste na aplicação de um método acompanhamento dos resultados e atingimento das 
metas baseado nos princípios do Ciclo PDCA, buscando a melhoria contínua do processo (Bartels et al., 2012; Erlandsson e Duhan, 2008; Araújo et al., 2016).

\section{INVESTIGAÇÃO DOS FATORES QUE CONTRIBUEM PARA O AUMENTO DO INVENTÁRIO}

A identificação do problema é necessária quando nos deparamos com um resultado indesejado em um processo. A complexidade dos sistemas de gestão do inventário, que envolvem grande quantidade de variáveis que afetam o resultado financeiro das empresas, reforça a necessidade da correta identificação e descrição do problema da obsolescência (Campos, 2009; Yin, 2015; Antony, 2006).

O time multifuncional é composto por um pequeno grupo de pessoas (três a cinco participantes - máximo dez) com habilidades complementares e comprometidas com um mesmo propósito, metas de desempenho e abordagem, com objetivo de proporcionar sinergia e maximizar os resultados (Latino et al., 2006).

A identificação das normas, procedimentos, políticas, questões regulatórias ou exigências de clientes que impactem a obsolescência do inventário é fundamental para identificar as variáveis e determinar o plano de ação. Entre as técnicas para levantamento destas questões estão as entrevistas com especialistas das áreas, brainstorming, diagrama de causa e efeito, método dos 5 porquês, mapeamento de processos, árvores de falha e diagramas lógicos (Latino et al., 2006).

Vários fatores que influenciam o risco de obsolescência do inventário (Chopra e Sodhi, 2004; Tang e Musa, 2011; Butt et al., 2015; Kessler e Brendel, 2016), os quais podem ser identificados através de entrevistas com especialistas das áreas, brainstorming, diagrama de causa e efeito, método dos 5 porquês, mapeamento de processos, árvores de falha ou diagramas lógicos (Latino et al., 2006; Kumar e Saranga, 2010; Kessler e Brendel, 2016).

\section{CLASSIFICACCÃO DO ESTOQUE DE PRODUTOS EM FAIXAS DE IDADE PARA MENSURAÇÃO DO RISCO DE OBSOLESCÊNCIA DO INVENTÁRIO}

A chave para uma análise bem-sucedida é certificar-se de que a equipe dispõe dos dados necessários para determinação das causas do problema (Latino et al., 2006). A coleta de dados deve ser precedida de um estudo inicial sobre o problema através de procedimentos explícitos e previamente planejados (Yin, 2015).

A classificação dos itens em diferentes categorias é necessária para aplicar as medidas mais adequadas para cada tipo ou classe de item (Boylan et al., 2008; Syntetos et al., 2010; Wu, 2013). A classificação do inventário pode ser realizada através dos parâmetros do sistema ERP da empresa ou do conhecimento de especialistas da área.

Apesar da escassa literatura acadêmica abordando o envelhecimento do inventário sob a ótica do risco de obsolescência (Chen et al., 2011; Wu, 2013; Li et al., 2014; Westerman, 2015; Raza et al., 2017), o estudo de caso realizado pelo 
autor obteve êxito na classificação do inventário em faixas de idade, com posterior determinação de medidas para redução do risco de obsolescência. Esta classificação pode ser feita com base nos registros contábeis de inventário, datas das ordens de produção e números de série dos produtos acabados.

\section{DEFINIÇÃO DO CONJUNTO DE VARIÁVEIS MAIS APROPRIADO PARA PROJEÇÃO DO INVENTÁRIO AO LONGO DO TEMPO}

O estudo prévio do processo e a definição das características relacionadas ao objetivo da análise são etapas importantes para o processo de planejamento e coleta de dados. É essencial que se desenvolva uma teoria antes de iniciar a coleta de dados para qualquer estudo de processo (Yin, 2015).

A coleta de dados isolados separa o fenômeno de seu contexto, tornando-se possível dedicar atenção especial àquelas variáveis julgadas de maior relevância. A qualidade dos dados e a relevância das variáveis é fundamental para garantir a eficácia das análises e a efetividade das ações (Yin, 2015).

A redução do número de variáveis conduz a um modelo de previsão mais adequado. Embora a seleção de variáveis possa ser feita de forma empírica, é recomendável que seja feita através de algoritmos apropriados (Gauchi e Chagnon, 2001; Guyon e Elisseeff, 2003; Balcaen e Ooghe, 2006; Andersen e Bro, 2010; Masiero e Anzanello, 2011).

Modelos de previsão são importantes para definir métodos de previsão de falhas e sistemas de alerta precoces, buscando direcionar ações apropriadas para evitar falhas, gerar informações úteis para a tomada de decisão e melhorar os resultados (Huang et al., 2005; Aziz e Dar, 2006; Ferrer et al., 2008; Huang et al., 2008; Boylan et al., 2008; Van Jaarsveld e Dekker, 2010; Syntetos et al., 2010; Wu, 2010; Bonney e Jaber, 2011; Wu, 2013; Jennings, 2015).

\section{DEFINIÇÃO DO CONJUNTO DE DIRETRIZES MAIS APROPRIADAS PARA REDUÇÃO DO INVENTÁRIO COM RISCO DE OBSOLESCÊNCIA}

Um estudo de caso é uma investigação empírica que investiga um fenômeno contemporâneo dentro de seu contexto real, especialmente quando os limites entre o fenômeno e o contexto não estão claramente definidos. Em posse de dados e informações detalhadas, e entendendo o conjunto de variáveis com maior impacto sobre o problema, reúnem-se os requisitos para correta definição do escopo do projeto (Yin, 2015).

As linhas de base são pontos de partida para medir a mudança de um determinado estado ou data, e são amplamente aceitos em diversas áreas de pesquisa. Os dados podem ser registrados em uma matriz contendo a linha de base, os níveis atuais de resultado, padrões de comparação, metas e tendências (Moldan et al., 2012, apud ten Brink, 2007; Therivel, 2012).

Os objetivos do projeto devem ser definidos de forma clara, sucinta, específica, realizável, realista e mensurável para que o resultado ou efeito indesejado de um processo seja eliminado (Antony, 2006; Santos e Miraglia, 2009; Campos, 2009; Yin, 2015). 
O plano de ação é necessário para coordenar e dar direção a um projeto, definindo onde se pretende chegar e o que se deseja alcançar, e deve ser tomado como guia e ser passível de replanejamento (Araújo et al., 2016). À medida em que as oportunidades de melhoria são identificadas é possível buscar resultados mais expressivos através da melhoria continua (Santos e Miraglia, 2009, apud Garcia e Amaral, 2005).

Quanto maior a prioridade do evento a ser analisado, mais rapidamente o processo de análise deve ser conduzido. É recomendável demonstrar compromisso através de reuniões periódicas agendadas com antecedência e realizadas de forma regular, para que os projetos logrem êxito em seus objetivos (Latino et al., 2006). As reuniões de revisão de resultados servem como mecanismo para identificar obstáculos e definir ações futuras, além de monitorar e divulgar o progresso (Antony, 2006).

\section{GESTÃO DA REDUÇ̃̃O DO RISCO DE OBSOLESCÊNCIA DO INVENTÁRIO}

O Ciclo PDCA pressupõe coleta periódica de dados em um ciclo contínuo para chegar a melhoria contínua dos resultados (Santos e Miraglia, 2009). O time multifuncional é responsável por providenciar, mensalmente, a coleta dos dados necessários.

A previsão é uma questão central na elaboração de estratégias, dada a presunção de que o que pode ser previsto pode ser controlado. A previsão possibilita o controle, permitindo escolher os meios apropriados para alcançar os resultados desejados, e contribui para que as empresas se mantenham na estratégia planejada através de uma visão de o futuro, conduzindo a resultados favoráveis (Wiltbank et al., 2006).

O ciclo PDCA é utilizado em problemas cujas causas e contramedidas são conhecidas pelas organizações. Através da aplicação do método, o problema será delimitado, uma meta será estabelecida, as causas serão identificadas e ações corretivas serão implantadas, de forma a eliminar o problema de forma definitiva (Melo e Caramori, 2001).

Uma atividade importante do PDCA é a padronização dos processos identificados pelas fases anterior que tiveram resultados satisfatórios, para que sejam novamente aplicados em situações similares, consolidando o processo de melhoria contínua (Santos e Miraglia, 2009).

O Ciclo PDCA parte do pressuposto da avaliação constante de todo o sistema como forma de detectar falhas, antecipando a ocorrência de eventos indesejados. Sua aplicação pressupõe um ciclo contínuo que busca a identificação dos pontos de melhoria do sistema, buscando a melhoria contínua dos resultados (Santos e Miraglia, 2009). 


\section{CARACTERIZAÇÃO DA EMPRESA}

Uma empresa fabricante de bens de consumo instalada no Brasil iniciou um projeto de redução de inventário por demanda de suas controladoras internacionais. Seu principal produto utiliza um elevado percentual de componentes importados, com ciclo de compras e produção de 150 dias e acentuada sazonalidade de vendas. Dada a capacidade limitada de produção, a empresa produz os produtos acabados para estoque com base na previsão de vendas. Os principais clientes desta empresa são grandes grupos varejistas, e a empresa enfrenta concorrência de outros fabricantes instalados no Brasil. O mercado exige atualizações tecnológicas e de design constantes, demandando a renovação da linha de produtos a cada dezoito meses.

A recente crise econômica afetou o mercado do principal produto da empresa. A conjunção da crise econômica com o período de baixa sazonalidade no início de 2015 e o ciclo de compras e produção de 150 dias conduziram a empresa a um cenário de inventário crescente. Como agravante, a empresa está sujeita a uma norma que atribui risco crescente de perda do inventário por obsolescência em função de sua idade, demandando a provisão em resultado de um percentual do valor do estoque com idade superior a 180 dias. Considerando o cenário de inventário crescente, o resultado contábil da empresa ao final do exercício restará prejudicado caso haja uma parcela significativa de estoque com idade superior a 180 dias.

Este cenário levou a empresa a definir um plano de metas para redução do inventário. O plano, porém, não foi suficiente para projetar a parcela de inventário que estaria acima de 180 dias ao final do ano, devido ao envelhecimento natural do inventário e devoluções de clientes. A análise do envelhecimento natural do inventário e sua contribuição sobre o inventário acima de 180 dias mostrou-se complexa, dada a incerteza da venda e alterações nas dinâmicas de mercado. A previsão das devoluções de produtos pelos clientes ou por problemas de transporte também se mostrou complexa, devido a imprevisibilidade dos problemas logísticos e do impacto da crise no comportamento dos clientes. Com base neste cenário, as controladoras internacionais da empresa exigiram um plano de ação de redução do inventário com metas a serem alcançadas até dezembro de 2015, com foco na redução do inventário acima de 180 dias e projeção do saldo de inventário sujeito a provisão, visando proteger o resultado anual.

\section{APLICAÇÃO DO MÉTODO}

\section{Identificação dos Fatores que Contribuem para o Aumento do Inventário}

Os fatores que contribuem para o aumento do inventário foram levantados pela direção e especialistas da empresa, sendo os principais: (i) a crise econômica e redução de demanda do mercado; (ii) o comportamento sazonal da venda, (iii) o ciclo de compras e produção de 150 dias; (iv) a devolução de produtos por 
clientes ou devido a sinistros de transporte, e; (v) a operação do FIFO (First In, First Out) nos armazéns.

O time multifuncional foi definido pelos Diretores e Gerentes das áreas de Operações e Comercial, e contou com 10 participantes das áreas de Produção, Logística, Finanças, Planejamento Industrial, Engenharia de Produtos, Comercial e Garantia e Pós-Vendas.

As regras e procedimentos aplicáveis a estoques foram obtidas através de brainstorming e entrevistas com especialistas. Dentre os fatores identificados, os de maior impacto no resultado foram: (i) a provisão para perda ou obsolescência para inventário com idade superior a 180 dias; (ii) a ausência de política de devoluções de clientes; (iii) a lógica de operação do FIFO no sistema WMS (Warehouse Management System), e; (iv) a ausência de política comercial para produtos descontinuados.

O valor da provisão para perda ou obsolescência para inventário com idade superior a 180 dias (prática imposta pelas controladoras internacionais da empresa) é definida em função da idade do inventário conforme o Quadro 4.

Quadro 4 - Classes de idade do inventário e provisão para perdas

\begin{tabular}{|l|c|c|c|c|c|c|}
\hline Idade do Inventário & $\begin{array}{c}\text { 0 a 30 } \\
\text { dias }\end{array}$ & $\begin{array}{c}31 \text { a 90 } \\
\text { dias }\end{array}$ & $\begin{array}{c}\text { 91 a 180 } \\
\text { dias }\end{array}$ & $\begin{array}{c}\text { 181 a } \\
\text { 360 dias }\end{array}$ & $\begin{array}{c}\text { 361 a } \\
\mathbf{7 2 0} \text { dias }\end{array}$ & $\begin{array}{c}\text { Mais de } \\
\text { 720 dias }\end{array}$ \\
\hline $\begin{array}{l}\text { Provisão para perdas e } \\
\text { obsolescência }\end{array}$ & $0 \%$ & $0 \%$ & $0 \%$ & $20 \%$ & $40 \%$ & $50 \%$ \\
\hline
\end{tabular}

Fonte: Dados da empresa (2015)

Quanto ao FIFO, verificou-se que os produtos devolvidos pelos clientes ou por sinistros de transporte, ao retornarem aos armazéns, eram interpretados pelo sistema WMS como um produto novo (recém-fabricado), visto que o sistema considera a data de entrada dos produtos no armazém (e não a data de fabricação) para efeitos de FIFO, conduzindo a um envelhecimento acima do esperado.

A identificação dos fatores que podem gerar estoque obsoleto foi realizada através de brainstorming e do método dos 5 porquês, e os fatores elencados pelo grupo como principais contribuintes foram: (i) o método de gestão do FIFO nos armazéns; (ii) o grande volume de devolução de clientes; (iii) a introdução de novos produtos antes de eliminar o estoque dos produtos anteriores; (iv) o descasamento de estoque de produtos modulares vendidos em conjunto; (v) a ausência de política de venda específica para produtos com baixo giro de estoque; (vi) a ausência de política de vendas para produtos descontinuados, e; (vii) a falta de gestão dos produtos devolvidos.

\section{Classificação do Estoque de Produtos em Faixas de Idade para Mensuração do Risco de Obsolescência do Inventário}

Os dados coletados do sistema ERP, dos reportes da área Financeira e dos indicadores das demais áreas envolvidas na análise estão listadas no Quadro 5, e compreendem o período de setembro de 2014 a abril de 2015. Seu conjunto foi definido como a porção de treino dos dados. 
Quadro 5 - Dados coletados pelo time multifuncional mensalmente.

\begin{tabular}{|c|c|c|}
\hline DADO & DEFINIÇÃO & OBJETIVO PRELIMINAR \\
\hline Venda do período & $\begin{array}{l}\text { Venda apurada em cada um dos } \\
\text { períodos (meses), aberta por itens, em } \\
\text { dólares sem impostos }\end{array}$ & $\begin{array}{l}\text { Avaliar a relação entre o volume } \\
\text { de vendas e o envelhecimento do } \\
\text { inventário }\end{array}$ \\
\hline $\begin{array}{l}\text { Venda de sucata de produtos } \\
\text { do período }\end{array}$ & $\begin{array}{l}\text { Venda de sucata de produtos em cada } \\
\text { um dos períodos (meses) em dólares } \\
\text { sem impostos }\end{array}$ & $\begin{array}{l}\text { Avaliar a performance da venda de } \\
\text { produtos sucateados }\end{array}$ \\
\hline $\begin{array}{l}\text { Volume de produção do } \\
\text { período }\end{array}$ & $\begin{array}{l}\text { Produção realizada em cada um dos } \\
\text { períodos (meses) em horas-padrão de } \\
\text { produção (CSH - Cost Standard } \\
\text { Hours) }\end{array}$ & $\begin{array}{l}\text { Avaliar a relação entre o volume } \\
\text { de produção e o envelhecimento do } \\
\text { inventário }\end{array}$ \\
\hline Produção do período & $\begin{array}{l}\text { Produção realizada em cada um dos } \\
\text { períodos (meses), aberta por itens, em } \\
\text { unidades }\end{array}$ & $\begin{array}{l}\text { Avaliar a relação entre o volume } \\
\text { de produção e o envelhecimento do } \\
\text { inventário }\end{array}$ \\
\hline $\begin{array}{l}\text { Produtos descasados na } \\
\text { produção }\end{array}$ & $\begin{array}{l}\text { Quantidade de produtos modulares } \\
\text { produzidos descasados no final de cada } \\
\text { um dos períodos (meses) em unidades }\end{array}$ & $\begin{array}{l}\text { Avaliar o impacto do } \\
\text { descasamento de produtos no } \\
\text { planejamento de produção e na } \\
\text { produção no envelhecimento do } \\
\text { inventário }\end{array}$ \\
\hline $\begin{array}{l}\text { Tempo médio de casamento } \\
\text { de produtos modulares }\end{array}$ & $\begin{array}{l}\text { Tempo médio entre a produção de } \\
\text { módulos complementares na produção } \\
\text { a cada período }\end{array}$ & $\begin{array}{l}\text { Avaliar o impacto do tempo } \\
\text { necessário para disponibilizar } \\
\text { todos os módulos necessários para } \\
\text { venda no envelhecimento do } \\
\text { inventário }\end{array}$ \\
\hline $\begin{array}{l}\text { Volume de retrabalho de } \\
\text { produtos no período }\end{array}$ & $\begin{array}{l}\text { Quantidade de produtos indisponíveis } \\
\text { para venda retrabalhados em cada um } \\
\text { dos períodos (meses) e disponibilizados } \\
\text { para venda }\end{array}$ & $\begin{array}{l}\text { Avaliar o impacto da performance } \\
\text { do retrabalho de produtos na } \\
\text { produção no envelhecimento do } \\
\text { inventário }\end{array}$ \\
\hline Inventário total por item & $\begin{array}{l}\text { Total de inventário do período por item } \\
\text { em dólares }\end{array}$ & $\begin{array}{l}\text { Avaliar o comportamento do } \\
\text { inventário ao longo do tempo }\end{array}$ \\
\hline $\begin{array}{l}\text { Inventário de produtos } \\
\text { acabados por faixa de idade }\end{array}$ & $\begin{array}{l}\text { Total de inventário de produtos } \\
\text { acabados aberto por item e por faixas } \\
\text { de idade em cada um dos períodos } \\
\text { (meses) em dólares }\end{array}$ & $\begin{array}{l}\text { Avaliar o ritmo em que o } \\
\text { inventário está envelhecendo ao } \\
\text { longo do tempo }\end{array}$ \\
\hline $\begin{array}{l}\text { Inventário de produtos } \\
\text { descasados }\end{array}$ & $\begin{array}{l}\text { Total de produtos descasados no } \\
\text { estoque em cada um dos períodos } \\
\text { (meses), aberto por item, em unidades }\end{array}$ & $\begin{array}{l}\text { Avaliar o impacto do } \\
\text { descasamento de produtos no } \\
\text { envelhecimento do inventário }\end{array}$ \\
\hline $\begin{array}{l}\text { Causas do descasamento do } \\
\text { estoque }\end{array}$ & $\begin{array}{l}\text { Identificação da causa raiz mais } \\
\text { provável de cada produto descasado no } \\
\text { estoque em cada um dos períodos } \\
\text { (meses) }\end{array}$ & $\begin{array}{l}\text { Avaliar as principais causas de } \\
\text { descasamento de estoque }\end{array}$ \\
\hline $\begin{array}{l}\text { Inventário de produtos } \\
\text { indisponíveis para venda }\end{array}$ & $\begin{array}{l}\text { Total de produtos indisponíveis para } \\
\text { venda no inventário em cada um dos } \\
\text { períodos (meses), aberto por item, em } \\
\text { dólares }\end{array}$ & $\begin{array}{l}\text { Avaliar o impacto do estoque de } \\
\text { produtos indisponíveis para venda } \\
\text { no envelhecimento do inventário }\end{array}$ \\
\hline $\begin{array}{l}\text { Causas do estoque de produtos } \\
\text { indisponíveis para venda }\end{array}$ & $\begin{array}{l}\text { Identificação da causa raiz mais } \\
\text { provável de cada produto indisponível } \\
\text { para venda no estoque em cada um dos } \\
\text { períodos (meses) }\end{array}$ & $\begin{array}{l}\text { Avaliar as principais causas de } \\
\text { produtos indisponíveis para venda } \\
\text { no estoque }\end{array}$ \\
\hline Performance do FIFO & $\begin{array}{l}\text { Percentual de itens faturados } \\
\text { corretamente, obedecendo às regras do } \\
\text { FIFO (lotes mais antigos primeiro) }\end{array}$ & $\begin{array}{l}\text { Avaliar se a Expedição está } \\
\text { cumprindo as regras de separação } \\
\text { de itens para faturamento seguindo } \\
\text { os critérios do FIFO }\end{array}$ \\
\hline $\begin{array}{l}\text { Devoluções de produtos } \\
\text { acabados }\end{array}$ & $\begin{array}{l}\text { Total de itens devolvidos } \\
\text { independentemente do motivo em cada } \\
\text { um dos períodos (meses) em dólares } \\
\text { sem impostos }\end{array}$ & $\begin{array}{l}\text { Avaliar a relação entre o volume } \\
\text { de devoluções e o envelhecimento } \\
\text { do inventário }\end{array}$ \\
\hline $\begin{array}{l}\text { Motivos de devoluções de } \\
\text { produtos acabados }\end{array}$ & $\begin{array}{l}\text { Identificação da causa raiz mais de } \\
\text { cada produto acabado devolvido para o } \\
\text { estoque em cada um dos períodos } \\
\text { (meses) }\end{array}$ & $\begin{array}{l}\text { Avaliar as principais causas de } \\
\text { devolução de produtos }\end{array}$ \\
\hline $\begin{array}{l}\text { Performance da introdução de } \\
\text { novos produtos }\end{array}$ & $\begin{array}{l}\text { Saldo de estoque de produtos } \\
\text { descontinuados após a introdução de } \\
\text { novos produtos, em dólares }\end{array}$ & $\begin{array}{l}\text { Avaliar a efetividade do } \\
\text { planejamento de introdução de } \\
\text { novos produtos }\end{array}$ \\
\hline
\end{tabular}

Fonte: Dados da empresa (2015)

A classificação dos itens em categorias foi necessária para interpretação precisa dos dados e definição dos métodos mais adequados de gestão para cada 
categoria. Originalmente, os itens foram classificados conforme o cadastro de itens do sistema ERP, exposto na Figura 2.

Figura 2 - Classificação original dos itens no sistema ERP

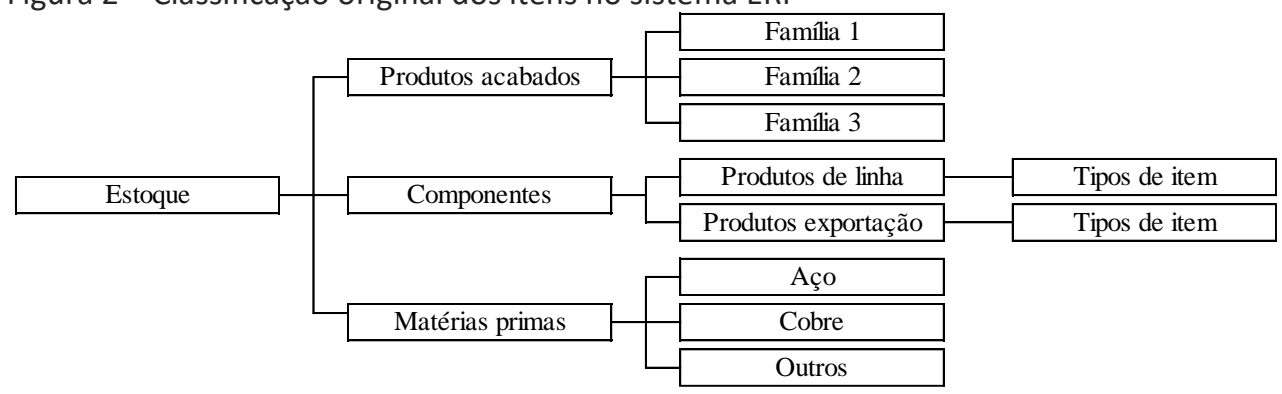

Fonte: Dados da empresa (2015)

Para a análise desejada, criou-se uma nova classificação em função da situação de cada item no inventário (com base no conhecimento de especialistas das áreas envolvidas no problema), exposta na Figura 3.

Figura 3 - Classificação original dos itens no sistema ERP

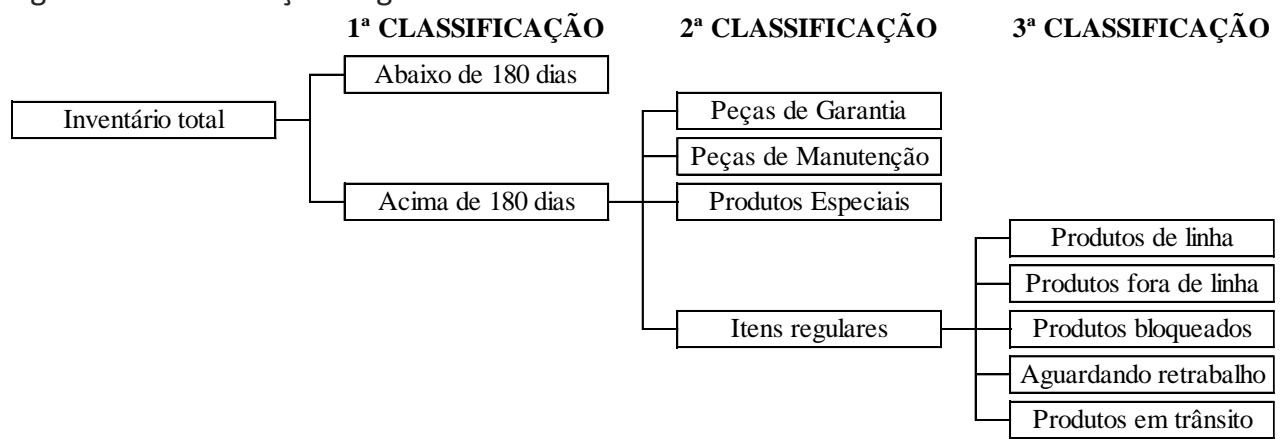

Fonte: Dados da empresa (2015)

O inventário foi classificado nas faixas de idade apresentadas no Quadro 4, para atender aos requisitos das controladoras internacionais quanto ao risco associado à idade do inventário e determinar medidas assertivas para redução do risco de obsolescência específicas para cada item e faixa de idade.

\section{Definição do Conjunto de Variáveis Mais Apropriado para Projeção do Inventário ao Longo do Tempo}

O time multifuncional realizou uma análise preliminar do processo a partir dos dados coletados, visando definir quais características do processo possuíam maior relevância no envelhecimento do inventário, estabelecer quais questões demandam maior profundidade de estudo e quais variáveis se mostram mais relevantes para o processo. O foco principal da análise foi o inventário de produtos acabados, que representava $69 \%$ do inventário total.

O conjunto de variáveis julgadas de maior relevância sobre o envelhecimento do inventário foi identificado pelo time multifuncional e especialistas das áreas. As primeiras análises foram feitas em Excel com regressão linear combinando diferentes variáveis, conforme o Quadro 6. 
Quadro 6 - Projeção do envelhecimento do estoque de produtos acabados através de regressão linear em Excel

\begin{tabular}{|c|c|c|c|}
\hline ANÁLISE & FERRAMENTA & VARIÁVEIS & $\begin{array}{c}\text { RESULTADO } \\
\left(\mathbf{R}^{2}\right)\end{array}$ \\
\hline 1 & Regressão linear & $\begin{array}{c}\mathrm{X} 1=\text { Venda do período } \\
\mathrm{X} 2=\text { Produção do período } \\
\mathrm{Y}=\text { Envelhecimento do inventário no período }\end{array}$ & 0,5857 \\
\hline 2 & Regressão linear & $\begin{array}{c}\text { X1 = Variação da venda (mês atual dividido pelo mês } \\
\text { anterior) } \\
\text { X2 = Variação da produção (mês atual dividido pelo mês } \\
\text { anterior) } \\
\begin{array}{c}\text { Y = Variação do envelhecimento do inventário (mês atual } \\
\text { dividido pelo mês anterior) }\end{array}\end{array}$ & 0,0962 \\
\hline 3 & Regressão linear & $\begin{array}{c}\text { X1 = Variação da venda (mês atual dividido pelo mês } \\
\text { anterior) } \\
\begin{array}{c}\text { X2 = Variação da produção (mês atual dividido pelo mês } \\
\text { anterior) } \\
\text { Y = Envelhecimento do inventário no período }\end{array}\end{array}$ & 0,0939 \\
\hline 4 & Regressão linear & $\begin{array}{c}\mathrm{X} 1=\text { Venda do período } \\
\mathrm{X} 2=\text { Produção do período } \\
\mathrm{Y}=\text { Variação do envelhecimento do inventário (mês atual } \\
\text { dividido pelo mês anterior) }\end{array}$ & 0,5906 \\
\hline 5 & Regressão linear & $\begin{array}{l}\text { X1 = Inventário abaixo de } 180 \text { dias no período } \\
\text { X2 = Produção do período } \\
\text { Y = Envelhecimento do inventário no período }\end{array}$ & 0,4303 \\
\hline 6 & Regressão linear & $\begin{array}{c}\text { X1 = Inventário abaixo de } 180 \text { dias no período } \\
\text { X1 = Variação da produção (mês atual dividido pelo mês } \\
\text { anterior) } \\
\begin{array}{c}\mathrm{Y}=\text { Variação do envelhecimento do inventário (mês atual } \\
\text { dividido pelo mês anterior) }\end{array}\end{array}$ & 0,3336 \\
\hline 7 & Regressão linear & $\begin{array}{c}\text { X1 = Inventário total do período } \\
\text { X2 = Produção do período } \\
\mathrm{Y}=\text { Envelhecimento do inventário no período }\end{array}$ & 0,6617 \\
\hline
\end{tabular}

Fonte: Dados da empresa (2015)

Após a análise com regressão linear, o time multifuncional realizou análises com mais de duas variáveis com o objetivo de obter resultados mais robustos. Duas novas variáveis foram introduzidas na análise, sendo (i) o inventário de produtos acabados com idade inferior a 180 dias e (ii) a performance do FIFO. 0 Quadro 7 mostra as diferentes combinações utilizadas para cálculo do impacto do envelhecimento do inventário de produtos acabados através de regressão múltipla. 
Quadro 7 - Projeção do envelhecimento do estoque de produtos acabados através de regressão múltipla em Excel

\begin{tabular}{|c|c|c|c|}
\hline ANÁLISE & FERRAMENTA & VARIÁVEIS & $\begin{array}{c}\text { RESULTADO } \\
\left(\mathbf{R}^{\mathbf{2}}\right)\end{array}$ \\
\hline 8 & Regressão múltipla & $\begin{array}{c}\text { X1 = Inventário total do período } \\
\text { X2 = Venda do período } \\
\text { X3 = Produção do período } \\
\text { X4 = Devoluções do período } \\
\text { Y = Variação do envelhecimento do inventário (mês } \\
\text { atual dividido pelo mês anterior) }\end{array}$ & 0,6577 \\
\hline 9 & Regressão múltipla & $\begin{array}{c}\mathrm{X} 1=\text { Inventário total do período } \\
\mathrm{X} 2=\text { Venda do período } \\
\mathrm{X} 3=\text { Produção do período } \\
\mathrm{X} 4=\text { Devoluções do período } \\
\mathrm{Y}=\text { Envelhecimento do inventário no período }\end{array}$ & 0,6689 \\
\hline 10 & Regressão múltipla & $\begin{array}{c}\text { X1 = Inventário acima de } 90 \text { dias no período } \\
\text { X2 = Venda do período } \\
\text { X3 = Produção do período } \\
\text { X4 = Devoluções do período } \\
\text { Y = Envelhecimento do inventário no período }\end{array}$ & 0,6493 \\
\hline 11 & Regressão múltipla & $\begin{array}{c}\text { X1 = Inventário abaixo de } 180 \text { dias no período } \\
\text { X2 = Venda do período } \\
\text { X3 = Produção do período } \\
\text { X4 = Devoluções do período } \\
\text { Y = Envelhecimento do inventário no período }\end{array}$ & 0,6690 \\
\hline 12 & Regressão múltipla & $\begin{array}{c}\text { X1 = Inventário abaixo de } 180 \text { dias no período } \\
\text { X2 = Venda do período } \\
\text { X3 = Produção do período } \\
\text { X4 = Devoluções do período } \\
\text { Y = Variação do envelhecimento do inventário (mês } \\
\text { atual dividido pelo mês anterior) }\end{array}$ & 0,6579 \\
\hline 13 & Regressão múltipla & $\begin{array}{c}\text { X1 = Inventário abaixo de } 180 \text { dias no período } \\
\text { X2 = Venda do período } \\
\text { X3 } 3=\text { Devoluções do período } \\
\text { Y = Envelhecimento do inventário no período }\end{array}$ & 0,6382 \\
\hline 14 & Regressão múltipla & $\begin{array}{c}\text { X1 = Inventário abaixo de } 180 \text { dias no período } \\
\text { X2 = Venda do período } \\
\text { X3 = Produção do período } \\
\text { Y = Envelhecimento do inventário no período }\end{array}$ & 0,6408 \\
\hline 15 & Regressão múltipla & $\begin{array}{c}\text { X1 = Inventário abaixo de } 180 \text { dias no período } \\
\text { X2 = Produção do período } \\
\text { X3 = Devoluções do período } \\
\text { Y = Variação do envelhecimento do inventário (mês } \\
\text { atual dividido pelo mês anterior) }\end{array}$ & 0,5256 \\
\hline 16 & Regressão múltipla & $\begin{array}{c}\text { X1 = Inventário abaixo de } 180 \text { dias no período } \\
\text { X2 = Venda do período } \\
\text { X3 = Produção do período } \\
\text { X4 = Devoluções do período } \\
\text { X5 = Performance do FIFO no período } \\
\text { Y = Envelhecimento do inventário no período }\end{array}$ & 0,8036 \\
\hline
\end{tabular}

Fonte: Dados da empresa (2015)

O melhor resultado foi obtido através de regressão múltipla combinando cinco variáveis para projeção do envelhecimento do inventário de produtos acabados, obtendo-se um $\mathrm{R}^{2}$ de 0,80 , conforme exposto no Quadro 8. 
Quadro8 - Dados utilizados na regressão múltipla da análise 16 do Quadro 9

\begin{tabular}{|c|c|c|c|c|c|c|}
\hline MÊS & $\begin{array}{c}\text { INVENTÁRIO } \\
<\mathbf{1 8 0} \text { DIAS } \\
(\mathbf{X 1})\end{array}$ & $\begin{array}{c}\text { VENDA } \\
(\mathbf{X 2})\end{array}$ & $\begin{array}{c}\text { PRODUÇÃo } \\
(\mathbf{X 3 3})\end{array}$ & $\begin{array}{c}\text { DEVOLUÇÕES } \\
(\mathbf{X 4 )}\end{array}$ & $\begin{array}{c}\text { FIFO } \\
(\mathbf{X 5})\end{array}$ & $\begin{array}{c}\text { ENVELHECIMENTO } \\
(\mathbf{Y})\end{array}$ \\
\hline set/14 & 107.445 & 98.357 & 377.491 & 3.577 & 0,67 & 2.012 \\
\hline out/14 & 106.988 & 110.052 & 404.900 & 1.955 & 0,67 & -1.943 \\
\hline nov/14 & 94.056 & 106.602 & 353.277 & 1.938 & 0,67 & 522 \\
\hline dez/14 & 68.877 & 102.230 & 255.309 & 2.307 & 0,67 & -2.820 \\
\hline jan/15 & 75.604 & 51.031 & 242.027 & 2.007 & 0,67 & 1.485 \\
\hline fev/15 & 68.231 & 64.811 & 218.947 & 1.940 & 0,67 & 607 \\
\hline mar/15 & 84.792 & 52.509 & 283.289 & 3.210 & 0,77 & 486 \\
\hline abr/15 & 103.965 & 37.502 & 235.986 & 6.230 & 0,80 & 3.188 \\
\hline mai/15 & 106.940 & 32.460 & 133.825 & 3.860 & 0,81 & 2.034 \\
\hline
\end{tabular}

Fonte: Dados da empresa (2015)

Com base nos dados acima, a análise por regressão múltipla forneceu as informações apresentadas no Quadro 9, que foram utilizadas para projeção dos resultados a partir de maio de 2015.

Quadro 9 - Resultados da análise de regressão múltipla com dados do Quadro 10 em Excel

RESUMO DOS RESULTADOS

\begin{tabular}{lr}
\hline \multicolumn{2}{c}{ Estatística de regressão } \\
\hline R múltiplo & 0,896 \\
R-Quadrado & 0,804 \\
R-quadrado & \\
ajustado & 0,476 \\
Erro padrão & 1394,8 \\
Observações & 9,000 \\
\hline
\end{tabular}

\begin{tabular}{lrrrrr} 
ANOVA & $g l$ & $S Q$ & $M Q$ & $F$ & $F$ de significação \\
\hline Regressão & 5 & 23877560 & 4775512 & 2,454 & 0,245 \\
Resíduo & 3 & 5836984 & 1945661 & & \\
Total & 8 & 29714544 & & & \\
& & & &
\end{tabular}

\begin{tabular}{lrrrrrrrr}
\hline & Coefic. & Erro padrão & Stat $t$ & valor-P & $\begin{array}{r}95 \% \\
\text { inferiores }\end{array}$ & $\begin{array}{r}95 \% \\
\text { superiores }\end{array}$ & $\begin{array}{r}\text { Inferior } \\
95,0 \%\end{array}$ & $\begin{array}{r}\text { Superior } \\
95,0 \%\end{array}$ \\
\hline Interseção & 19121 & 13633 & 1,402 & 0,255 & $-24268,3$ & 62510,5 & $-24268,3$ & 62510,5 \\
Variável X 1 & 0,062 & 0,049 & 1,263 & 0,296 & $-0,094$ & 0,218 & $-0,094$ & 0,218 \\
Variável X 2 & $-0,074$ & 0,036 & $-2,057$ & 0,132 & $-0,188$ & 0,040 & $-0,188$ & 0,040 \\
Variável X 3 & 0,001 & 0,012 & 0,077 & 0,943 & $-0,038$ & 0,040 & $-0,038$ & 0,040 \\
Variável X 4 & 0,631 & 0,600 & 1,053 & 0,370 & $-1,278$ & 2,541 & $-1,278$ & 2,541 \\
Variável X 5 & -29384 & 19917 & $-1,475$ & 0,237 & $-92770,4$ & 34001,8 & $-92770,4$ & 34001,8 \\
\hline
\end{tabular}

Fonte: Dados da empresa (2015)

O coeficiente de determinação $R^{2}$ obtido através da correlação múltipla se mostrou satisfatório $(0,8035)$, porém, o coeficiente $R^{2}$ ajustado $(0,4761)$ apresentou diferença relevante, indicando a necessidade de redução do número de variáveis para obtenção de um modelo de previsão mais rápido, com menor complexidade e melhores propriedades estatísticas. Para tal, o time multifuncional realizou uma nova análise utilizando o Minitab, inicialmente, com os dados codificados, para tornar a definição do conjunto de variáveis mais robusta, conforme o Quadro 10. 
Quadro 10 - Dados codificados utilizados na regressão múltipla em Minitab.

\begin{tabular}{|c|c|c|c|c|c|c|}
\hline MÊS & $\begin{array}{c}\text { INVENTÁRIO } \\
\text { <180 DIAS } \\
\text { (C1) }\end{array}$ & $\begin{array}{l}\text { VENDA } \\
\text { (C2) }\end{array}$ & $\begin{array}{l}\text { PRODUÇÃO } \\
\text { (C3) }\end{array}$ & $\begin{array}{c}\text { DEVOLUÇÕES } \\
\text { (C4) }\end{array}$ & $\begin{array}{c}\text { FIFO } \\
\text { (C5) }\end{array}$ & $\begin{array}{l}\text { ENVELHECIMENTO } \\
(\mathrm{Y})\end{array}$ \\
\hline $\mathrm{set} / 14$ & 1,00 & 1,00 & 1,00 & $-1,00$ & $-1,00$ & $-0,70$ \\
\hline out $/ 14$ & 0,30 & 0,90 & 0,70 & $-1,00$ & $-1,00$ & 0,10 \\
\hline nov/14 & $-1,00$ & 0,80 & 0,20 & $-0,80$ & $-1,00$ & $-1,00$ \\
\hline $\mathrm{dez} / 14$ & $-0,60$ & $-0,50$ & 0,10 & $-1,00$ & $-1,00$ & 0,40 \\
\hline $\mathrm{jan} / 15$ & $-1,00$ & $-0,20$ & 0,00 & $-1,00$ & $-1,00$ & 0,10 \\
\hline $\mathrm{fev} / 15$ & $-0,20$ & $-0,50$ & 0,30 & $-0,40$ & 0,30 & 0,10 \\
\hline $\mathrm{mar} / 15$ & 0,80 & $-0,90$ & 0,10 & 1,00 & 0,80 & 1,00 \\
\hline abr/15 & 1,00 & $-1,00$ & $-0,50$ & $-0,10$ & 1,00 & 0,60 \\
\hline mai/15 & 0,10 & $-0,90$ & $-1,00$ & $-0,90$ & 0,50 & $-0,30$ \\
\hline
\end{tabular}

Fonte: Dados da empresa (2015)

O resultado da análise realizada em Minitab com os dados do Quadro 10 são mostrados no Quadro 11 Figura 4.

Quadro 11 - Resultados da análise de regressão múltipla com dados codificados do Quadro 10 em Minitab

\begin{tabular}{|c|c|c|c|c|c|}
\hline \multicolumn{6}{|c|}{$\begin{array}{l}\text { Regression Analysis: } Y \text { versus C1; C2; C3; C4; C5 } \\
\text { The regression equation is } \\
Y=-0,022+0,359 C 1-0,988 C 2+0,245 C 3+0,526 C 4-0,722 C 5\end{array}$} \\
\hline Predictor & Coef & SE Coef & $\mathrm{T}$ & \multicolumn{2}{|l|}{$\mathrm{P}$} \\
\hline Constant & $-0,022$ & 0,271 & $-0,08$ & \multicolumn{2}{|l|}{0,938} \\
\hline $\mathrm{C} 1$ & 0,359 & 0,247 & 1,45 & \multicolumn{2}{|l|}{0,221} \\
\hline C2 & $-0,988$ & 0,392 & $-2,52$ & \multicolumn{2}{|l|}{0,065} \\
\hline C3 & 0,245 & 0,513 & 0,48 & \multicolumn{2}{|l|}{0,658} \\
\hline C4 & 0,526 & 0,358 & 1,47 & \multicolumn{2}{|l|}{0,216} \\
\hline C5 & $-0,722$ & 0,431 & $-1,68$ & \multicolumn{2}{|l|}{0,169} \\
\hline$S=0,405282$ & \multicolumn{3}{|c|}{$\mathrm{R}-\mathrm{Sq}=80,7 \%$} & \multicolumn{2}{|c|}{$R-S q(a d j)=56,6 \%$} \\
\hline \multicolumn{6}{|c|}{ Analysis of Variance } \\
\hline Source & DF & SS & MS & $\mathrm{F}$ & $\mathrm{P}$ \\
\hline Regression & 5 & 2,752 & 0,550 & 3,350 & 0,132 \\
\hline Residual Error & 4 & 0,657 & 0,164 & & \\
\hline Total & 9 & 3,409 & & & \\
\hline Source & DF & Seq SS & & & \\
\hline $\mathrm{C} 1$ & 1 & 0,554 & & & \\
\hline C2 & 1 & 0,989 & & & \\
\hline C3 & 1 & 0,663 & & & \\
\hline C4 & 1 & 0,085 & & & \\
\hline $\begin{array}{l}\text { C5 } \\
\text { No unusual ob }\end{array}$ & 1 & 0,461 & & & \\
\hline
\end{tabular}

Fonte: Dados da empresa (2015) 
Figura 4 - Residuais da análise de regressão múltipla com dados codificados do Quadro 10 em Minitab.
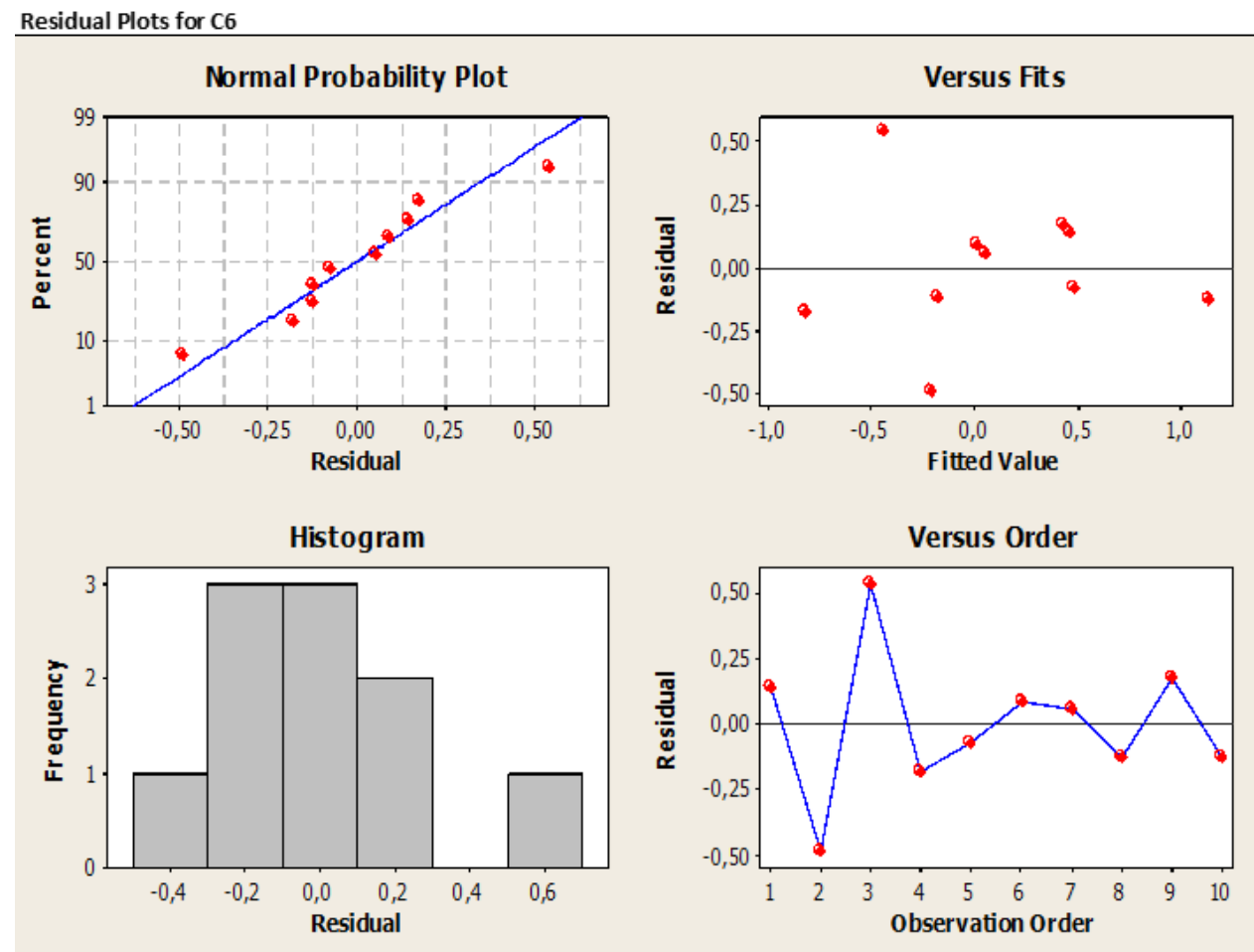

Fonte: Dados da empresa (2015)

Observou-se na análise em Minitab uma diferença relevante entre os coeficientes $R^{2}(0,807)$ e $R^{2}$ ajustado $(0,566)$, indicando a inclusão indiscriminada de variáveis. A redução das variáveis foi obtida utilizando regressão PLS com o método Best Subset Selection, utilizando o $\mathrm{R}^{2}$ ajustado e critério $\mathrm{Cp}$ de Mallow. $\mathrm{O}$ resultado desta análise é apresentado no Quadro 12, que mostra que o melhor resultado é obtido através da utilização de quatro variáveis (C1, C2, C4 e C5), com melhores resultados de $R^{2}(0,796)$ e $R^{2}$ ajustado $(0,633)$.

Quadro 12 - Resultados da análise através da função Best Subsets Selection em Minitab

\begin{tabular}{|c|c|c|c|c|c|c|c|c|c|}
\hline \multicolumn{10}{|c|}{ Best Subsets Regression: $\mathrm{Y}$ versus $\mathrm{C} 1 ; \mathrm{C} 2$; C3; C4; C5 } \\
\hline \multicolumn{10}{|c|}{ Response is $Y$} \\
\hline Mallows & & & & & $\mathrm{C}$ & $\mathrm{C}$ & $\mathrm{C}$ & $\mathrm{C}$ & $\mathrm{C}$ \\
\hline Vars & $\mathrm{R}-\mathrm{Sq}$ & R-Sq(adj) & $\mathrm{Cp}$ & & 1 & 2 & 3 & 4 & 5 \\
\hline 1 & 46,5 & 39,8 & 5,1 & 0,478 & & & & $x$ & \\
\hline 1 & 28,7 & 19,8 & 8,8 & 0,551 & & $x$ & & & \\
\hline 2 & 59,5 & 47,9 & 4,4 & 0,444 & & $x$ & $x$ & & \\
\hline 2 & 53,4 & 40,1 & 5,7 & 0,476 & & $x$ & & $x$ & \\
\hline 3 & 65,6 & 48,4 & 5,1 & 0,442 & & $x$ & $x$ & $x$ & \\
\hline 3 & 65,2 & 47,8 & 5,2 & 0,445 & & $x$ & & $x$ & $x$ \\
\hline 4 & 79,9 & 63,3 & 4,2 & 0,373 & $x$ & $x$ & & $x$ & $x$ \\
\hline 4 & 70,6 & 47,1 & 6,1 & 0,448 & & $x$ & $x$ & $x$ & $x$ \\
\hline 5 & 80,7 & 56,6 & 6,0 & 0,405 & $x$ & $x$ & $x$ & $x$ & $x$ \\
\hline
\end{tabular}

Fonte: Dados da empresa (2015)

A partir da definição do conjunto ideal de variáveis, uma nova análise foi feita em Minitab utilizando os dados reais não codificados das variáveis C1, C2, C4 e C5, cujos resultados estão apresentados nos Quadros 13 e 14 e Figura 5. 
Quadro 13 - Dados reais utilizados na regressão múltipla após a aplicação da ferramenta Best Subsets do Minitab

\begin{tabular}{|c|c|c|c|c|c|}
\hline MÊS & $\begin{array}{c}\text { INVENTÁRIO } \\
\text { <180 DIAS (C1) }\end{array}$ & $\begin{array}{c}\text { VENDA } \\
\text { (C2) }\end{array}$ & $\begin{array}{c}\text { DEVOLUÇÕES } \\
\text { (C4) }\end{array}$ & $\begin{array}{c}\text { FIFO } \\
\text { (C5) }\end{array}$ & $\begin{array}{c}\text { ENVELHECIMENTO } \\
\text { (Y) }\end{array}$ \\
\hline $\mathrm{set} / 14$ & 107.445 & 98.357 & 3.577 & 0,67 & 2.012 \\
\hline $\mathrm{out} / 14$ & 106.988 & 110.052 & 1.955 & 0,67 & -1.943 \\
\hline $\mathrm{nov} / 14$ & 94.056 & 106.602 & 1.938 & 0,67 & 522 \\
\hline $\mathrm{dez} / 14$ & 68.877 & 102.230 & 2.307 & 0,67 & -2.820 \\
\hline $\mathrm{jan} / 15$ & 75.604 & 51.031 & 2.007 & 0,67 & 1.485 \\
\hline $\mathrm{fev} / 15$ & 68.231 & 64.811 & 1.940 & 0,67 & 607 \\
\hline $\mathrm{mar} / 15$ & 84.792 & 52.509 & 3.210 & 0,77 & 486 \\
\hline $\mathrm{abr} / 15$ & 103.965 & 37.502 & 6.230 & 0,80 & 3.188 \\
\hline $\mathrm{mai} / 15$ & 106.940 & 32.460 & 3.860 & 0,81 & 2.034 \\
\hline
\end{tabular}

Fonte: Dados da empresa (2015)

Quadro 14 - Resultados da análise de regressão múltipla com dados reais do Quadro 13

\begin{tabular}{|c|c|c|c|c|c|}
\hline \multicolumn{6}{|c|}{$\begin{array}{l}\text { Regression Analysis: } Y \text { versus } \mathbf{C 1} \text {; C2; C4; C5 } \\
\text { The regression equation is } \\
Y=22050+0,058 \mathrm{C} 1-0,069 \mathrm{C} 2+0,893 \mathrm{C} 4-34365 \mathrm{C5}\end{array}$} \\
\hline Predictor & Coef & SE Coef & $\mathrm{T}$ & $P$ & \\
\hline Constant & 22050 & 10284 & 2,14 & 0,085 & \\
\hline $\mathrm{C} 1$ & 0,058 & 0,034 & 1,70 & 0,151 & \\
\hline $\mathrm{C2}$ & $-0,069$ & 0,025 & $-2,72$ & 0,042 & \\
\hline C4 & 0,893 & 0,404 & 2,21 & 0,078 & \\
\hline C5 & -34365 & 14487 & 2,37 & 0,064 & \\
\hline$S=1168,30$ & \multicolumn{2}{|c|}{$R-S q=78,3 \%$} & \multicolumn{3}{|c|}{$R-S q(a d j)=61,0 \%$} \\
\hline \multicolumn{6}{|c|}{ Analysis of Variance } \\
\hline Source & DF & SS & MS & $\mathrm{F}$ & $\mathrm{P}$ \\
\hline Regression & 4 & 24654612 & 6163653 & 4,520 & 0,065 \\
\hline Residual Error & 5 & 6824629 & 1364926 & & \\
\hline Total & 9 & 31479242 & & & \\
\hline Source & DF & Seq SS & & & \\
\hline $\mathrm{C} 1$ & 1 & 4635614 & & & \\
\hline $\mathrm{C} 2$ & 1 & 8914616 & & & \\
\hline C4 & 1 & 3424321 & & & \\
\hline C5 & 1 & 7680061 & & & \\
\hline
\end{tabular}

Fonte: Dados da empresa (2015) 
Figura 5 - Residuais da análise de regressão múltipla com dados reais do Quadro 13 Residual Plots for C5
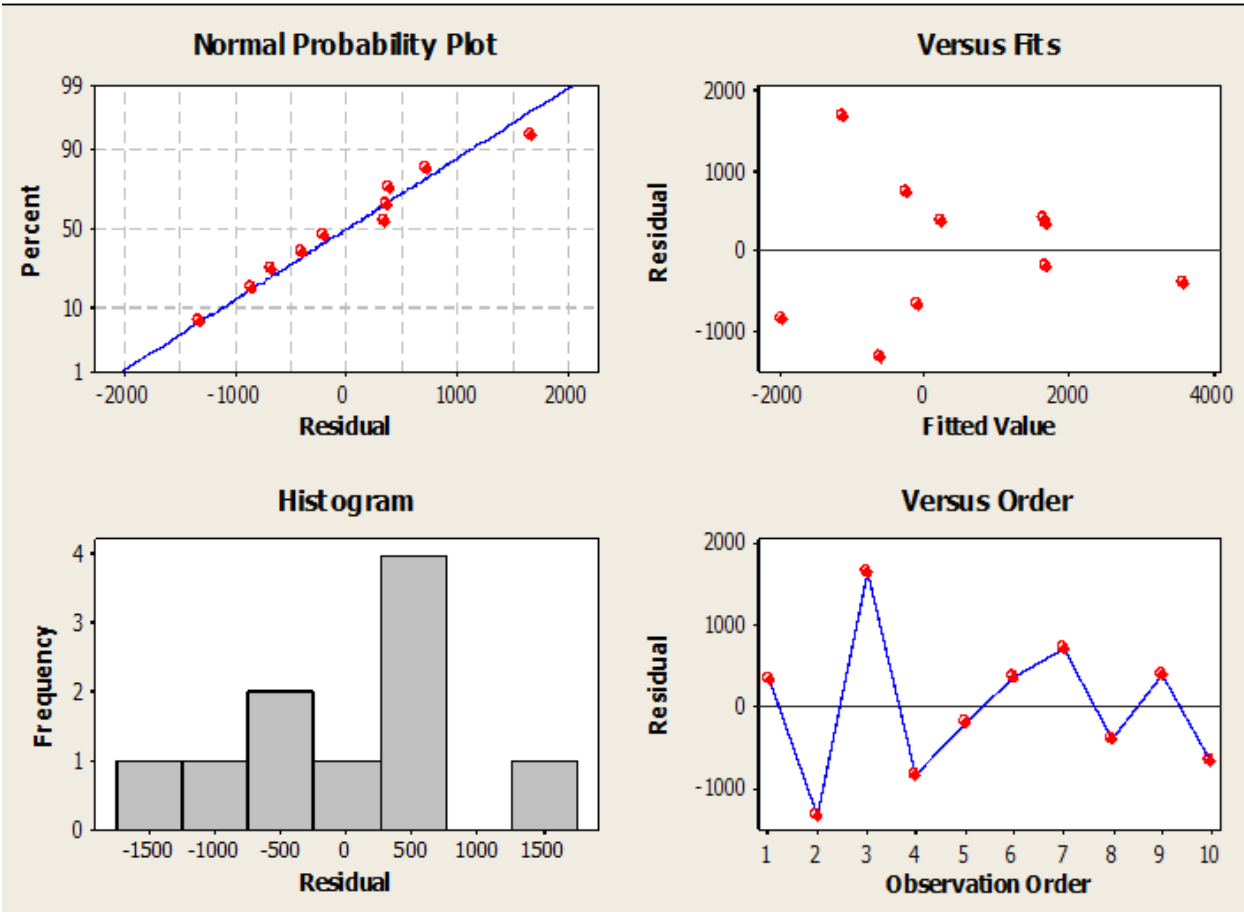

Fonte: Dados da empresa (2015)

O modelo de previsão utilizado para desenvolver alertas precoces e direcionar ações apropriadas para evitar o envelhecimento do inventário e reduzir o risco de obsolescência é apresentado na equação (18).

$$
c_{5}=22050+0,0583 c_{1}+0,0692 c_{2}+0,893 c_{3}-34365 c_{4}
$$

Para a projeção dos resultados futuros foram definidas premissas para: (i) vendas (última previsão de vendas da empresa, em dólares); (ii) produção (última previsão de produção da empresa, em horas de produção); (iii) inventário menor do que 180 dias (última previsão de inventário da empresa, em dólares, aplicando sobre ele todas as ações já endereçadas de redução do inventário até dezembro); (iv) devoluções (mapeamento e projeção das devoluções futuras, em dólares, com base no conhecimento de especialistas da área), e; (v) FIFO (projeção de melhoria da performance do FIFO com base na experiência de especialistas da área, considerando os planos de ação já implantados).

Os resultados projetados do envelhecimento do inventário de produtos acabados para os meses de junho a dezembro utilizando as previsões e premissas acima são apresentados no Quadro 15. 
Quadro 15 - Projeção do envelhecimento do inventário de produtos acabados através de regressão múltipla em Minitab

\begin{tabular}{|c|c|c|c|c|c|}
\hline MÊS & $\begin{array}{c}\text { INVENTÁRIO } \\
\text { <180 DIAS (C1) }\end{array}$ & $\begin{array}{c}\text { VENDA } \\
\text { (C2) }\end{array}$ & $\begin{array}{c}\text { DEVOLUÇÕES } \\
\text { (C3) }\end{array}$ & $\begin{array}{c}\text { FIFO } \\
\text { (C4) }\end{array}$ & $\begin{array}{c}\text { ENVELHECIMENTO } \\
(\text { Y) }\end{array}$ \\
\hline jun/15 & 90.325 & 36.841 & 2.165 & 0,78 & -64 \\
\hline jul/15 & 92.022 & 36.946 & 4.747 & 0,82 & 979 \\
\hline $\mathrm{ago} / 15$ & 124.419 & 61.608 & 4.000 & 0,84 & -168 \\
\hline $\mathrm{set} / 15$ & 133.095 & 100.428 & 4.000 & 0,83 & -2.005 \\
\hline out/15 & 125.084 & 119.753 & 4.000 & 0,85 & -4.445 \\
\hline nov/15 & 99.957 & 132.520 & 4.000 & 0,84 & -6.618 \\
\hline dez/15 & 83.310 & 114.219 & 4.000 & 0,84 & -6.274 \\
\hline
\end{tabular}

Fonte: Dados da empresa (2015)

A projeção do estoque ao longo do tempo foi utilizada para priorizar as ações e alocar recursos de forma eficaz para redução do risco de obsolescência do inventário. Conhecendo o comportamento futuro de determinado grupo de itens a partir das variáveis observadas e da projeção dos resultados ao longo do tempo, foi possível definir ações assertivas e específicas para cada tipo ou grupo de item.

\section{Definição do Conjunto de Diretrizes Mais Apropriadas para Redução do Inventário com Risco de Obsolescência}

De posse dos dados e informações detalhadas, e conhecendo o conjunto de variáveis com maior impacto sobre o envelhecimento e risco de obsolescência do inventário, a empresa definiu como escopo a redução do inventário acima de 180 dias em $60 \%$ até dezembro de 2015 , visando proteger o resultado contábil através da redução da provisão para perda e obsolescência.

O ponto de partida para medir a performance das ações e definir a base para futuras previsões de impacto e monitoramento foi a posição do inventário contábil da empresa em 30 de abril de 2015.

Os objetivos do projeto foram definidos pela empresa de forma clara, sucinta, específica, realizável, realista e mensurável, com base em dados e sugestões de especialistas das áreas. Todas as áreas envolvidas no problema receberam ações a serem implantadas e monitoradas sob sua responsabilidade.

As ações definidas foram reunidas em um único plano de ação, acompanhado mensalmente nas reuniões periódicas de acompanhamento do projeto. O comparativo entre o previsto e o realizado de cada uma das ações era pauta das reuniões de trabalho do grupo multifuncional.

Foi definido um calendário de reuniões com datas fixas divididas em três grupos, sendo: (i) reuniões do grupo de trabalho multifuncional com a participação esporádica de convidados das áreas envolvidas (semanal); (ii) reuniões de comunicação interna para divulgar os resultados obtidos à alta gestão da empresa no Brasil (mensal), e; (iii) reuniões de comunicação externa, para divulgar os resultados obtidos à controladora no exterior e/ou ao conselho diretor (mensal). 
O time multifuncional foi responsável por providenciar a coleta de dados de estoque e das variáveis selecionadas, em um ciclo contínuo mensal. Para garantir a acurácia das informações, as fontes e os métodos para obtenção dos dados foram padronizados.

O time multifuncional foi responsável por projetar o valor do estoque ao longo do tempo para cada faixa de idade, incluindo a cada mês as coletas de dados mais recentes, em um ciclo contínuo mensal.

A metodologia PDCA foi utilizada para coletar dados, avaliar resultados, verificar a eficácia e definir novas ações mensalmente, buscando a melhoria contínua dos resultados. Para tal, a primeira reunião do grupo de trabalho multifuncional de cada mês era dedicada à análise dos dados coletados e verificação da eficácia das ações no mês anterior, a segunda reunião tratava a projeção do estoque ao longo do tempo e avaliação do risco de obsolescência do estoque para cada grupo de item e/ou faixa de idade, na terceira reunião definiam-se novas ações, e a quarta reunião de cada mês era destinada a preparação da apresentação das reuniões de comunicação interna e externa.

As melhorias implementadas que conduziram a uma redução do risco de obsolescência do inventário foram padronizadas e implantadas nas rotinas de trabalho da empresa (preferencialmente, incluídas no sistema ERP).

Após a revisão dos resultados e projeção do estoque, as ações previamente definidas foram revisadas mensalmente e alteradas sempre que necessário, de forma a garantir o atingimento dos resultados.

\section{CONCLUSÃO}

A projeção do inventário acima de 180 dias, sob a ótica dos gestores da empresa e de especialistas da área de gestão de inventário e custos industriais, é coerente com os valores esperados e com o comportamento do inventário ao longo dos anos. Observa-se que no mês de julho o envelhecimento do inventário deve continuar acontecendo, porém em ritmo menor, e que a partir de agosto o aumento expressivo do faturamento aliado à melhora da performance do FIFO leva a uma redução do envelhecimento do inventário.

A utilização da regressão múltipla se mostrou plenamente eficaz na resolução do problema proposto. No início de julho, após a atualização dos dados do fechamento contábil de junho observou-se que o envelhecimento do inventário de produtos acabados aconteceu dentro do intervalo esperado pelas análises de regressão, e que até dezembro as variações entre o previsto e o realizado não ultrapassaram $5 \%$.

Para efeitos de padronização da análise, sugere-se que a rotina de verificar planejado vs. realizado ocorra mensalmente. Além disso, mensalmente as variáveis deem ser revisadas com as últimas previsões da empresa para refinar ainda mais as projeções futuras. Os gestores da empresa, bem como as controladoras internacionais, entenderam que a solução proposta é adequada para este tipo de projeção. 
Um ponto importante a ser observado é que a variável C3 (volume de produção), a qual através da função "Best Subsets" do Minitab foi eliminada da análise, é de fácil obtenção na indústria em geral, e contribui para o resultado final da análise agregando apenas uma variável adicional. Embora esta variável, não tenha se mostrado relevante no caso em questão, pode ser importante para outras empresas. Desta forma, como critério do pesquisador, pode-se optar por manter esta variável em futuras análises, com vistas a aplicar este modelo em outras empresas ou indústrias. 


\title{
Approach for Predictive Variable Selection in the Context of Inventory Control
}

\begin{abstract}
Inventory obsolescence is a prominent phenomenon in organizations, demanding the use of methods that identify excessive inventory before it becomes obsolete. This paper proposes a method to select independent variables in predictive regression models for the projection of inventory over time, in order to reduce the risk of future obsolescence. The proposed approach has five steps, the first four of which are dedicated to identifying the factors that contribute to inventory obsolescence, inventory classification into categories and age ranges, selection of variables in PLS context, regression modeling for projection of the age of the inventory over time and the definition of guidelines for reducing the risk of obsolescence. The fifth step of the method uses the concept of the PDCA cycle seeking continuous improvement of process and results. In the application in a consumer goods industry, the method accurately predicted more than $95 \%$ the amount of inventory per age range over a six-month horizon.
\end{abstract}

KEYWORDS: Stock Management. Obsolescence. Selection of Variables. PLS. Regression Analysis. 


\section{REFERÊNCIAS}

AGRESTI, A.; KATERI, M. Categorical data analysis. Berlin/Heidelberg: Springer, 2011.

ALGHAZZAWI, A.; LENNOX, B. Model predictive control monitoring using multivariate statistics. Journal of Process Control, 19(2), 314-327, 2009. crossref

ANDERSEN, C. M.; BRO, R. Variable selection in regression - a tutorial. Journal of Chemometrics, 24(11-12), 728-737, 2010. crossref

ANTONY, J. Six sigma for service processes. Business Process Management Journal, 12(2), 234-248, 2006. crossref

ANZANELLO, M. J. Seleção de variáveis para classificação de bateladas produtivas com base em múltiplos critérios. Production Journal, 23(4), 858-865, 2013.

crossref

ANZANELLO, M. J.; ALBIN, S. L.; CHAOVALITWONGSE, W. A. Selecting the best variables for classifying production batches into two quality levels. Chemometrics and Intelligent Laboratory Systems, 97(2), 111-117, 2009. crossref

ARAÚJO, M. G.; QUEIROZ, J. V.; LIMA, J. C. F.; FERNANDES, G.; QUEIROZ, F. C. B. P.; FURUKAVA, M. Aplicação do ciclo PDCA na elaboração de um plano estratégico e implementação da Curva $A B C$ como ferramenta de suporte para o gerenciamento de estoques de uma distribuidora de alimentos hospitalares. Revista Espacios, Vol. 37 (№ 13), 2016.

AYRES, M.; AYRES JÚNIOR, M.; AYRES, D. L.; SANTOS, A. D. A. Aplicações estatísticas nas áreas das ciências bio-médicas. Instituto Mamirauá, Belém, 2007.

AZIZ, M. A.; DAR, H. A. Predicting corporate bankruptcy: where we stand? Corporate Governance: The international Journal of Business in Society, 6(1), 1833, 2006. crossref

BAKKE, H. A.; LEITE, A. D. M.; SILVA, L. D. Estatística multivariada: aplicação da análise fatorial na engenharia de produção. Revista Gestão Industrial, 4(4), 1-14, 2008. crossref

BAKKER, M.; RIEZEBOS, J.; TEUNTER, R. H. Review of inventory systems with deterioration since 2001. European Journal of Operational Research, 221(2), 275-284, 2012. crossref 
BALCAEN, S.; OOGHE, H. 35 years of studies on business failure: an overview of the classic statistical methodologies and their related problems. The British Accounting Review, 38(1), 63-93, 2006. crossref

BARTELS, B.; ERMEL, U.; SANDBORN, P.; PECHT, M. G. Strategies to the Prediction, Mitigation and Management of Product Obsolescence. 1 $1 \mathrm{a}$ ed. Hoboken, New Jersey (USA): John Wiley \& Sons Inc., 2012. crossref

BATTINI, D.; PERSONA, A.; SGARBOSSA, F. A. Sustainable EOQ model: theoretical formulation and applications. International Journal of Production Economics, 149, 145-153, 2014. crossref

BEAMON, B. M. Supply chain design and analysis: Models and methods. International Journal of Production Economics, 55(3), 281-294, 1998. crossref

BEAMON, B. M. Measuring supply chain performance. International Journal of Operations \& Production Management, 19(3), 275-292, 1999. crossref

BLUM, A. L.; LANGLEY, P. Selection of relevant features and examples in machine learning. Artificial Intelligence, 97(1), 245-271, 1997. crossref

BONNEY, M.; JABER, M. Y. Environmentally responsible inventory models: Nonclassical models for a non-classical era. International Journal of Production Economics, 133(1), 43-53, 2011. crossref

BOYLAN, J. E.; SYNTETOS, A. A.; KARAKOSTAS, G. C. Classification for forecasting and stock control: a case study. Journal of the Operational Research Society, 59(4), 473-481, 2008. crossref

BROSOFSKE, K. D.; FROESE, R. E.; FALKOWSKI, M. J.; BANSKOTA, A. A review of methods for mapping and prediction of inventory attributes for operational forest management. Forest Science, 60(4), 733-756, 2014. crossref

BUTT, T. E.; CAMILLERI, M.; PAUL, P.; JONES, K. G. Obsolescence types and the built environment - definitions and implications. International Journal of Environment and Sustainable Development, 14(1), 20-39, 2015. crossref

CAMPOS, J. C. Os impactos da conjuntura macroeconômica sobre o comércio no Brasil: uma análise empírica das grandes varejistas. Fundação Getúlio Vargas, Rio de Janeiro, 2009. 
CERVO, V. L.; ANZANELLO, M. J. Seleção de variáveis para clusterização de bateladas produtivas através de ACP e remapeamento kernel. Production, v. 25, n. 4, 2015. crossref

CHAE, B. Developing key performance indicators for supply chain: an industry perspective. Supply Chain Management: An International Journal, 14(6), 422428,2009 . crossref

CHEN, H.; RAMNATH, S.; RANGAN, S.; ROCK, S. Inventory write-downs in the semiconductor industry. The Fourth Accounting Research Conference, December 19-20, Gachibowli, Hyderabad, India, 2011.

CHONG, I. G.; JUN, C. H. Performance of some variable selection methods when multicollinearity is present. Chemometrics and Intelligent Laboratory Systems, 78(1), 103-112, 2005. crossref

CHOPRA, S.; SODHI, M. S. Managing risk to avoid supply-chain breakdown. MIT Sloan Management Review, 46(1), 53, 2004.

CLAASSEN, M. J.; VAN WEELE, A. J.; VAN RAAIJ, E. M. Performance outcomes and success factors of vendor managed inventory (VMI). Supply Chain Management: An International Journal, 13(6), 406-414, 2008. crossref

DASH, M.; LIU, H. Consistency-based search in feature selection. Artificial Intelligence, 151(1), 155-176, 2003. crossref

ERLANDSSON, B.; DUHAN, J. A Pragmatic Approach to Inventory Management. 2008

FERRER, A.; AGUADO, D.; VIDAL-PUIG, S.; PRATS, J. M.; ZARZO, M. PLS: A versatile tool for industrial process improvement and optimization. Applied Stochastic Models in Business and Industry, 24(6), 551-567, 2008. crossref

FISHER, M. L. What is the right supply chain for your product. Operations Management: Critical Perspectives on Business and Management, 4, 73, 2003.

GAUCHI, J. P.; CHAGNON, P. Comparison of selection methods of explanatory variables in PLS regression with application to manufacturing process data. Chemometrics and Intelligent Laboratory Systems, 58(2), 171-193, 2001. crossref 
GNANA, D. A. A.; APPAVU, S.; LEAVLINE, E. J. Literature Review on Feature Selection Methods for High-Dimensional Data. Methods, 136(1), 2016.

GUYON, I.; ELISSEEFF, A. An introduction to variable and feature selection. The Journal of Machine Learning Research, 3, 1157-1182, 2003.

HAIR, J. F.; BLACK, W. C.; BABIN, B. J.; ANDERSON, R. E.; AND TATHAM, R. L. Multivariate Data Analysis Seventh Edition. Pearson Education, New Jersey, 2009.

HENSELER, J.; RINGLE, C. M.; SINKOVICS, R. R. The use of partial least squares path modeling in international marketing. Advances in International Marketing, 20(1), 277-319, 2009.

HÖSKULDSSON, A. Variable and subset selection in PLS regression. Chemometrics and intelligent laboratory systems, 55(1), 23-38, 2001. crossref

HUANG, S. M.; TSAI, C. F.; YEN, D. C.; CHENG, Y. L. A hybrid financial analysis model for business failure prediction. Expert Systems With Applications, 35(3), 1034-1040, 2008. crossref

HUANG, W.; NAKAMORI, Y.; WANG, S. Y. Forecasting stock market movement direction with support vector machine. Computers \& Operations Research, 32(10), 2513-2522, 2005. crossref

ICHII, K.; TAKAHASHI, K.; MURAMATSU, R. Integrated production, inventory and distribution systems. The International Journal Of Production Research, v. 26, n. 3 , p. 473-482, 1988. crossref

JAMES, G.; WITTEN, D.; HASTIE, T.; TIBSHIRANI, R. An introduction to statistical learning (Vol. 6). New York: Springer, 2013. crossref

JANSSEN, L.; CLAUS, T.; SAUER, J. Literature review of deteriorating inventory models by key topics from 2012 to 2015. International Journal of Production Economics, 182, 86-112, 2016. crossref

JENNINGS, C. P. Forecasting obsolescence risk and product lifecycle with machine learning. Graduate Theses and Dissertations, 14825, lowa State University, 2015.

JOHNSON, R. A.; WICHERN, D. W. Applied multivariate statistical analysis, 6a ed. Upper Saddle River, NJ: Prentice Hall, 2007 
KASZNAR, I. K.; GONÇALVES, B. M. L. Regressão múltipla: uma digressão sobre seus usos. Instituto Business Consultoria Internacional, Rio de Janeiro, nd, 20p, 2011.

KAUFMANN, L.; GAECKLER, J. A structured review of partial least squares in supply chain management research. Journal of Purchasing and Supply Management, 21(4), 259-272, 2015. crossref

KESSLER, T.; BRENDEL, J. Planned Obsolescence and Product-Service Systems: Linking Two Contradictory Business Models. Journal of Competence-based Strategic Management, 8, 29-52, 2016.

KETTANEH, N.; BERGLUND, A.; WOLD, S. PCA and PLS with very large data sets. Computational Statistics \& Data Analysis, 48(1), 69-85, 2005. crossref

KHOLOPANE, P. The Effect of the Implementation of Six Sigma in Reducing Obsolete Stock and Controlling Stock Inventory at a Flavor Manufacturing Company: A Case Study. In: Proceedings of the International Multi Conference of Engineers and Computer Scientists (Vol. 2), 2016.

KRAJČOVIČ, M.; PLINTA, D. Comprehensive approach to the inventory control system improvement. Management and Production Engineering Review, 3(3), 34-44, 2012.

KUMAR, U. D.; SARANGA, H. Optimal selection of obsolescence mitigation strategies using a restless bandit model. European Journal of Operational Research, 200(1), 170-180, 2010. crossref

LATINO, R. J.; LATINO, K. C.; LATINO, M. A. Root cause analysis: improving performance for bottom-line results, 3a ed. Boca Rarton, FL: CRC Press, 2006. crossref

LI, L.; SHEN, C.; WANG, L.; ZHENG, L.; JIANG, Y.; TANG, L.; TANG, J. iMiner: mining inventory data for intelligent management. In: Proceedings of the $23 \mathrm{rd}$ ACM International Conference on Conference on Information and Knowledge Management, p. 2057-2059. ACM, 2014.

LILAND, K. H. Multivariate methods in metabolomics-from pre-processing to dimension reduction and statistical analysis. TrAC Trends in Analytical Chemistry, 30(6), 827-841, 2011. crossref 
LIU, H.; YU, L. Toward integrating feature selection algorithms for classification and clustering. IEEE Transactions on Knowledge and Data Engineering, 17(4), 491-502, 2005. crossref

MÁRIO, P. D. C. Contribuição ao estudo da solvência empresarial: uma análise de modelos de previsão-estudo exploratório aplicado em empresas mineiras (Doctoral dissertation, Universidade de São Paulo), 2002.

MASIERO, M. S.; ANZANELLO, M. J. Seleção de variáveis para predição utilizando regressão linear em processos logísticos de distribuição. Universidade Federal do Rio Grande do Sul, 2011.

MATEOS-APARICIO, G. Partial least squares (PLS) methods: Origins, evolution, and application to social sciences. Communications in Statistics-Theory and Methods, 40(13), 2305-2317, 2011. crossref

MEHMOOD, T.; LILAND, K. H.; SNIPEN, L.; SABB $\varnothing$, S. A review of variable selection methods in partial least squares regression. Chemometrics and Intelligent Laboratory Systems, 118, 62-69, 2012. crossref

MELO, A. S.; HEPP, L. U. Ferramentas estatísticas para análises de dados provenientes de biomonitoramento. Oecologia Brasiliensis, 12(3), 8, 2008.

MELO, C. P.; CARAMORI, E.J. PDCA Método de melhorias para empresas de manufatura - versão 2.0. Belo Horizonte: Fundação de Desenvolvimento Gerencial, 2001.

MENG, X.; THÖRNBERG, B.; OLSSON, L. Strategic proactive obsolescence management model. IEEE Transactions on Components, Packaging and Manufacturing Technology, 4(6), 1099-1108, 2014. crossref

MILETIC, I.; QUINN, S.; DUDZIC, M.; VACULIK, V.; CHAMPAGNE, M. An industrial perspective on implementing on-line applications of multivariate statistics. Journal of Process Control, 14(8), 821-836, 2004. crossref

MINGOTI, S. A. Análise de dados através de métodos de estatística multivariada: uma abordagem aplicada. Belo Horizonte: UFMG, 2005.

MOLDAN, B.; JANOUŠKOVÁ, S.; HÁK, T. How to understand and measure environmental sustainability: Indicators and targets. Ecological Indicators, 17, 413, 2012. cross ref 
MONTGOMERY, D. C. Design and analysis of experiments, $5 \underline{a}$ ed. John Wiley \& Sons, 2008.

MUNDSTOCK, P. Relação entre planejamento estratégico e desempenho superior. Universidade Federal do Rio Grande do Sul, 2008.

NAKAGAWA, S.; SCHIELZETH, H. A general and simple method for obtaining $\mathrm{R}^{2}$ from generalized linear mixed-effects models. Methods in Ecology and Evolution, 4(2), 133-142, 2013. crossref

NENES, G.; PANAGIOTIDOU, S.; TAGARAS, G. Inventory management of multiple items with irregular demand: A case study. European Journal of Operational Research, 205(2), 313-324, 2010. crossref

ORELIEN, J. G.; EDWARDS, L. J. Fixed-effect variable selection in linear mixed models using $R^{2}$ statistics. Computational Statistics \& Data Analysis, 52(4), 18961907, 2008. crossref

PANIGRAHI, A. K. Relationship between inventory management and profitability: An empirical analysis of Indian cement companies. Asia Pacific Journal of Marketing \& Management Review, 2(7), 2013.

PINÇE, C.; DEKKER, R. An inventory model for slow moving items subject to obsolescence. European Journal of Operational Research, 213(1), 83-95, 2011. crossref

PINGLE, P. Selection of obsolescence resolution strategy based on a multi criteria decision model (Doctoral dissertation, lowa State University), 2015.

PORTER, M. E. Vantagem competitiva: criando e sustentando um desempenho superior. Rio de Janeiro: Campus, 1992.

POURAKBAR, M.; FRENK, J. B. G.; DEKKER, R. End-of-Life Inventory Decisions for Consumer Electronics Service Parts. Production and Operations Management, 21(5), 889-906, 2012. crossref

RAZA, B.; KARIM, U.; HUSSAIN, D. Inventory Aging: An Impediment to the Value Chain - A Case Related to the Automotive Industry, 2017.

RENCHER, A. C. Methods of multivariate analysis. John Wiley \& Sons, 2003. 
ROSIPAL, R.; KRÄMER, N. Overview and recent advances in partial least squares. Lecture notes in Computer Science, v. 3940, p. 34, 2006. crossref

ROY, P. P.; ROY, K. On some aspects of variable selection for partial least squares regression models. QSAR \& Combinatorial Science, 27(3), 302-313, 2008. crossref

SAEYS, Y.; INZA, I.; LARRAÑAGA, P. A review of feature selection techniques in bioinformatics. Bioinformatics, 23(19), 2507-2517, 2007. crossref

SANDBORN, P.; PRABHAKAR, V.; AHMAD, O. Forecasting electronic part procurement lifetimes to enable the management of DMSMS obsolescence. Microelectronics Reliability, 51(2), 392-399, 2011. crossref

SANTOS, E. A. D.; MIRAGLIA, S. G. E. K. Arquivos abertos e instrumentos de gestão da qualidade como recursos para a disseminação da informação científica em segurança e saúde no trabalho. Ciência da Informação, 2009. crossref

SCHIELZETH, H. Simple means to improve the interpretability of regression coefficients. Methods in Ecology and Evolution, 1(2), 103-113, 2010. crossref

SINGH, R. K.; GARG, S. K.; DESHMUKH, S. G. Strategy development by SMEs for competitiveness: a review. Benchmarking: An International Journal, 15(5), 525547, 2008.

SONG, J. S.; ZIPKIN, P. Inventory control in a fluctuating demand environment. Operations Research, 41(2), 351-370, 1993. crossref

SONG, J. S.; ZIPKIN, P. H. Managing inventory with the prospect of obsolescence. Operations Research, 44(1), 215-222, 1996. crossref

STEIN, M.; ANZANELLO, M. J.; KAHMANN, A. Sistemática para identificação das variáveis preditivas mais relevantes em um processo do setor metal-mecânico. Revista Gestão Industrial, 10(1), 2014.

SYNTETOS, A. A.; BABAI, M. Z.; DAVIES, J.; STEPHENSON, D. Forecasting and stock control: A study in a wholesaling context. International Journal of Production Economics, 127(1), 103-111, 2010. crossref

TABACHNICK, B. G.; FIDELL, L. S. Using multivariate statistics, 5a ed. Boston: Allyn \& Bacon, 2007. 
TABAKHI, S.; MORADI, P.; AKHLAGHIAN, F. An unsupervised feature selection algorithm based on ant colony optimization. Engineering Applications of Artificial Intelligence, 32, 112-123, 2014. crossref

TANG, O.; MUSA, S. N. Identifying risk issues and research advancements in supply chain risk management. International Journal of Production Economics, 133(1), 25-34, 2011. crossref

TEUNTER, R. H.; SYNTETOS, A. A.; BABAI, M. Z. Intermittent demand: Linking forecasting to inventory obsolescence. European Journal of Operational Research, 214(3), 606-615, 2011. crossref

THERIVEL, R. Strategic environmental assessment in action. Routledge, 2012. crossref

THUMMALAPALLI, R. A Methodology to evaluate obsolete inventory in health care. University of Nebraska - Lincoln, 2010.

VAN DONK, D. P.; VAN DER VAART, T. A case of shared resources, uncertainty and supply chain integration in the process industry. International Journal of Production Economics, 96(1), 97-108, 2005. crossref

VAN JAARSVELD, W.; DEKKER, R. Estimating obsolescence risk from demand data to enhance inventory control - A case study. International Journal of Production Economics, 133(1), 423-431, 2011. crossref

VICINI, L.; SOUZA, A. M. Análise multivariada da teoria à prática. Santa Maria: UFSM, CCNE, 2005.

WANKE, P. Metodologia para gestão de estoques de peças de reposição: um estudo de caso em empresa brasileira. Revista Tecnologística, 60-65, 2005.

WESTERMAN, W. Working capital management programs: Yesterday, today, tomorrow. Journal of Modern Accounting and Auditing, 11(4), 210-217, 2015. crossref

WILD, T. Best practice in inventory management. Routledge, 2007. crossref

WILTBANK, R.; DEW, N.; READ, S.; SARASVATHY, S. D. What to do next? The case for non-predictive strategy. Strategic Management Journal, 27(10), 981-998, 2006. crossref 
WOLD, S.; SJÖSTRÖM, M.; ERIKSSON, L. PLS-regression: a basic tool of chemometrics. Chemometrics and Intelligent Laboratory Systems, 58(2), 109130, 2001. crossref

WONG, C. Y.; ARLBJ ØRN, J. S.; HVOLBY, H. H.; JOHANSEN, J. Assessing responsiveness of a volatile and seasonal supply chain: A case study. International Journal of Production Economics, 104(2), 709-721, 2006. crossref

WU, J. Z. Inventory write-down prediction for semiconductor manufacturing considering inventory age, accounting principle, and product structure with real settings. Computers \& Industrial Engineering, 65(1), 128-136, 2013. crossref

WU, W. W. Beyond business failure prediction. Expert Systems with Applications, 37(3), 2371-2376, 2010. crossref

YANG, Y.; WILLIAMS, E. Logistic model-based forecast of sales and generation of obsolete computers in the US. Technological Forecasting and Social Change, 76(8), 1105-1114, 2009. crossref

YIN, R. K. Estudo de Caso: Planejamento e Métodos. Bookman Editora, 2015.

YU, L.; LIU, H. Efficient feature selection via analysis of relevance and redundancy. The Journal of Machine Learning Research, 5, 1205-1224, 2004.

ZHANG, L.; YUAN, Z.; BI, J. Predicting future quantities of obsolete household appliances in Nanjing by a stock-based model. Resources, Conservation and Recycling, 55(11), 1087-1094, 2011. crossref

ZIMMER, J. Seleção de variáveis preditivas com base em índices de importância das variáveis e regressão PLS. Universidade Federal do Rio Grande do Sul, 2012.

ZIMMER, J.; ANZANELLO, M. J. Um novo método para seleção de variáveis preditivas com base em índices de importância. Produção, São Paulo, 24, 84-93, 2014. 
Recebido: 29 jan 2018

Aprovado: 29 set. 2018

DOI: 10.3895/gi.v14n4.7690

Como citar:

BURGEL, E.; ANZANELLO, M.J. Abordagem para Seleção de Variáveis Preditivas no Contexto de

Controle de Inventários. R. Gest. Industr., Ponta Grossa, v. 14, n. 4, p. 154-195, out./dez. 2018.

Disponível em: $\leq$ https://periodicos.utfpr.edu.br/rgi>. Acesso em: XXX.

Correspondência:

Evandro Burgel

Rua Engenheiro Edmundo Gardolinski, número 115, Casa 1, Bairro Boa Vista, Porto Alegre, Rio Grande do

Sul, Brasil. Mestrando em Engenharia de Produção pela UFRGS. Telefone (51) 3209-7713. E-mail eburgel0512@gmail.com.

Direito autoral: Este artigo está licenciado sob os termos da Licença Creative Commons-Atribuição 4.0 Internacional.

\section{(c) (1)}

